\title{
Titanocene(III) chloride mediated radical induced addition-elimination route to the synthesis of racemic and optically active trisubstituted tetrahydrofurans: Formal synthesis of magnofargesin and $7^{\prime}$-epimagnofargesin
}

\author{
P CHAKRABORTY ${ }^{\mathrm{a}}$, S K MANDAL ${ }^{\mathrm{b}}$ and S C ROY ${ }^{\mathrm{a}, *}$

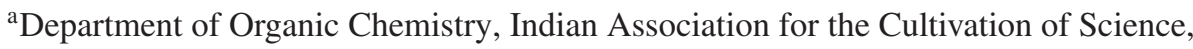 \\ Jadavpur, Kolkata 700 032, India \\ ${ }^{b}$ Department of Chemistry, Saldiha College, Saldiha, Bankura 722 173, India \\ e-mail: ocscr@iacs.res.in
}

MS received 25 February 2016; revised 19 April 2016; accepted 26 April 2016

\begin{abstract}
Titanocene(III) Chloride mediated radical induced synthesis of 4-benzylidene substituted tetrahydrofuran, a typical lignan skeleton, has been accomplished in good yield through addition-elimination route in racemic as well as in optically active forms. The method has been applied to the synthesis of furano lignans, magnofargesin (1) and 7'-epimagnofargesin (2) in optically active forms.
\end{abstract}

Keywords. Titanocene(III) chloride; radical; tetrahydrofurans; synthesis; furano lignans.

\section{Introduction}

Radical induced addition-elimination process has been used as a tool for the synthesis of bioactive natural products due to mild and simple reaction conditions along with a vast substrate tolerance. The early report ${ }^{1}$ by Kharasch et al., has further been developed ${ }^{2}$ by Heiba and Dessau describing an unexpected cascade radical cyclization initiated by intermolecular addition of carbon-centered trichloromethyl radical to alkynes. Interestingly, Baldwin et al., synthesized ${ }^{3}$ the cyclopentanoid isonitrile, the core skeleton of antibiotic metabolites of fungi in the genus trichoderma, via a novel radical addition-elimination method. Harris and Weiler ${ }^{4}$ prepared stereospecific exocyclic alkene by a consecutive radical cyclization-elimination process in 1987. In the same year, Pattenden and his group developed ${ }^{5}$ a cobalt-mediated addition-elimination protocol for the synthesis of carbon-carbon double bond. Baichi and Bosch $^{6}$ used the identical protocol for the synthesis of bicyclic $\beta$-lactams. Naito et al., successfully applied the addition-elimination process for asymmetric synthesis of (-)- $\alpha$-Kainic acid $^{7 \mathrm{a}}$ and a concise formal synthesis of (-)-martinellic acid. ${ }^{7 \mathrm{~b}}$ Some strained functionalized alkylidene-cyclobutanes ${ }^{8 \mathrm{a}}$ and fused

*For correspondence spirocyclic imines ${ }^{8 b}$ were also prepared using this technique. Banwell used this technique as a key step for the synthesis of aromatic erythrina alkaloids ${ }^{9 a}$ and chemoenzymatic approach towards the total synthesis of (+)-Brunsvigine. ${ }^{9 b}$ Such a useful technique in the field of radical chemistry has neither been cultivated nor used extensively ${ }^{10}$ by using titanocene(III) species as a radical initiator specially for the synthesis of natural products. ${ }^{11 \mathrm{a}, \mathrm{b}}$ Herein, we depict a novel methodology for the synthesis of benzylidene substituted tetrahydrofurans using $\mathrm{Cp}_{2} \mathrm{TiCl}$ induced radical based additionelimination strategy that has been implemented to the total synthesis of two furano lignans, magnofargesin (1) and its isomer $7^{\prime}$-epimagnofargesin (2). ${ }^{11 \mathrm{c}}$ The radical initiator titanocene(III) chloride $\left(\mathrm{Cp}_{2} \mathrm{TiCl}\right)$ was prepared from commercially available $\mathrm{Cp}_{2} \mathrm{TiCl}_{2}$ and $\mathrm{Zn}$ dust in THF under argon atmosphere. ${ }^{12}$

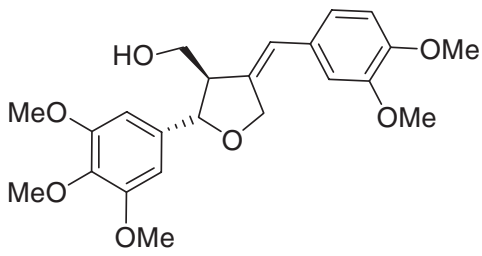

Magnofargesin (1)

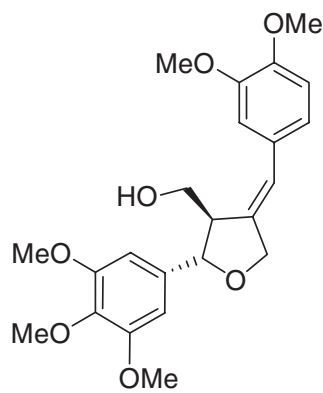

7'-Epimagnofargesin (2) 


\section{Experimental}

${ }^{1} \mathrm{H}$ NMR were recorded in $\mathrm{CDCl}_{3}$ on 300,400 and 500 $\mathrm{MHz}$ and ${ }^{13} \mathrm{C}$ NMR were recorded on 75, 100 and 125 $\mathrm{MHz}$ spectrometer respectively using TMS as an internal standard. IR spectra were recorded on a Shimadzu FTIR-8300 instrument. High-resolution mass spectra (HRMS) were obtained using a Qtof Micro YA263 instrument. Ethyl acetate was dried over anhydrous calcium chloride. Petroleum ether of boiling range 60$80^{\circ} \mathrm{C}$ and diethyl ether were dried over sodium. Silica gel of 60-120 mesh was used for column chromatography. THF used for radical cyclisation was super dried by distilling twice with sodium. DCM solvent was used after freshly distilling over $\mathrm{P}_{2} \mathrm{O}_{5}$. All the reactions were carried out either in argon or nitrogen atmosphere with oven-dried glass apparatus. Most of the compounds described are already reported in the literature and are characterized by NMR, IR and MASS spectral studies and have been compared with authentic samples.

Allylic alcohols 4a-g ${ }^{13}$ and the epoxides 5a-g ${ }^{14 c, 15-17}$ were prepared following the standard literature procedures.

\subsection{Preparation of the epoxy ether $\mathbf{6} \boldsymbol{a}$}

To a stirred suspension of $\mathrm{NaH}$ (53 mg, 50\% dispersion, $1.1 \mathrm{mmol})$ in dry THF $(1 \mathrm{~mL})$ was added dropwise a solution of epoxy alcohol $\mathbf{5 a}(75 \mathrm{mg}, 0.50 \mathrm{mmol})$ in dry THF $(5 \mathrm{~mL})$ at $0^{\circ} \mathrm{C}$ under nitrogen. After the liberation of hydrogen gas ceased (approx. $25 \mathrm{~min}$ ), a solution of dibromo compound 12 (166 mg, $0.60 \mathrm{mmol}$ ) in dry THF $(7.5 \mathrm{~mL})$ was added dropwise at $0^{\circ} \mathrm{C}$ over $10 \mathrm{~min}$. The reaction mixture was stirred at RT for $3 \mathrm{~h}$ and then carefully quenched with ice water. After removal of most of the solvent under reduced pressure, the resulting residue was extracted with diethyl ether $(4 \times 25 \mathrm{~mL})$. The combined ether extract was successively washed with water $(2 \times 10 \mathrm{~mL})$ and brine $(1 \times 10 \mathrm{~mL})$ and finally dried $\left(\mathrm{Na}_{2} \mathrm{SO}_{4}\right)$. Solvent was removed under reduced pressure and the crude mass obtained was purified by column chromatography over silica gel to furnish 2-(-2-bromo3-phenylallyloxy)(phenyl)methyl)oxirane (6a, $146 \mathrm{mg}$, $85 \%)$ as a viscous liquid and as an inseparable mixture of two isomers in approx. 3:1 ratio. IR (Neat): 3058, 2854, 1598, 1490, 1257, 1068, 756, $694 \mathrm{~cm}^{-1}$; ${ }^{1} \mathrm{H}$ NMR $\left(400 \mathrm{MHz}, \mathrm{CDCl}_{3}\right): \delta 2.66(\mathrm{dd}, J=2.4$, $4.8 \mathrm{~Hz}, 0.75 \mathrm{H}), 2.75-2.83(\mathrm{~m}, 1.25 \mathrm{H}), 3.23-3.24(\mathrm{~m}$, $0.25 \mathrm{H}), 3.27-3.30(\mathrm{~m}, 0.75 \mathrm{H}), 4.21-4.52$ (complex multiplets, $2.75 \mathrm{H}), 4.64(\mathrm{~d}, J=4.4 \mathrm{~Hz}, 0.25 \mathrm{H}), 7.29-7.43$ (m, $10 \mathrm{H}$ (aromatic hydrogens) $+1 \mathrm{H}$ (olefinic hydrogen); ${ }^{13} \mathrm{C} \mathrm{NMR}\left(75 \mathrm{MHz}, \mathrm{CDCl}_{3}\right): \delta 44.3,45.2,54.2$,
$55.0,55.2,57.1,79.4,81.9,84.8,84.9,86.5,86.7$, $122.6,122.7,127.2,127.4,127.7,128.2,128.3,128.5$, 128.6, 128.8, 129.1, 131.8, 137.4; HRMS: calcd. for $\mathrm{C}_{18} \mathrm{H}_{17} \mathrm{BrO}_{2}[\mathrm{M}+\mathrm{Na}]^{+}$367.0304; found: 367.0300 .

Compounds 6b-6g were prepared following the similar procedure used for the preparation of $\mathbf{6 a}$.

\subsection{2-(-2-Bromo-3-phenylallyloxy)(4-chlorophenyl) methyl)oxirane $(\boldsymbol{6} \boldsymbol{b})$}

Viscous liquid as an inseparable mixture of two isomers in approx. 1.5:1 ratio. Yield $88 \%$. IR (Neat): 3055, 1596, 1488, 1257, 1078, 1014, 756, $690 \mathrm{~cm}^{-1}$; ${ }^{1} \mathrm{H} \mathrm{NMR}\left(400 \mathrm{MHz}, \mathrm{CDCl}_{3}\right): \delta 2.62-2.65(\mathrm{~m}, 0.60 \mathrm{H})$, 2.72-2.78 (m, 1H), 2.81-2.84 (m, 0.40H), 3.18-3.26 (m, $1 \mathrm{H}), 4.20-4.52$ (complex multiplets, $2.60 \mathrm{H}), 4.60$ (d, $J=6 \mathrm{~Hz}, 0.40 \mathrm{H}), 7.28-7.44$ (m, 9H (aromatic hydrogens) $+1 \mathrm{H}$ (olefinic hydrogen); ${ }^{13} \mathrm{C}$ NMR $(75 \mathrm{MHz}$, $\left.\mathrm{CDCl}_{3}\right): \delta 44.28,45.3,54.0,54.8,57.2,57.3,78.9,81.0$, 84.5, 84.6, 86.8, 87.0, 122.5, 122.6, 128.4, 128.6, 128.8, 128.9, 129.0, 129.1, 131.8, 131.9, 134.5, 135.9; HRMS: calcd. for $\mathrm{C}_{18} \mathrm{H}_{16} \mathrm{BrClO}_{2}[\mathrm{M}+\mathrm{Na}]^{+}$400.9914; found, 400.9912 .

\subsection{2-(-2-Bromo-3-phenylallyloxy)(p-tolyl)methyl) oxirane $(\mathbf{6 c})$}

Viscous liquid as an inseparable mixture of two isomers in approx. 2:1 ratio. Yield 84\%. IR (Neat): 2921, 1514, 1490, 1263, 1076, 756, $692 \mathrm{~cm}^{-1} ;{ }^{1} \mathrm{H}$ NMR (500 $\left.\mathrm{MHz} \mathrm{CDCl}_{3}\right): \delta 2.36(2 \mathrm{~s}$ merged, $3 \mathrm{H}), 2.63-2.65(\mathrm{~m}$, $0.67 \mathrm{H}), 2.74-2.82(\mathrm{~m}, 1.33 \mathrm{H}), 3.21-3.23(\mathrm{~m}, 0.33 \mathrm{H})$, 3.26-3.29 (m, 0.67H), 4.14-4.48 (complex multiplets, $2.67 \mathrm{H}), 4.61(\mathrm{~d}, J=4.5 \mathrm{~Hz}, 0.33 \mathrm{H}), 7.10-7.63(\mathrm{~m}$, $9 \mathrm{H}$ (aromatic hydrogens) $+1 \mathrm{H}$ (olefinic hydrogen); ${ }^{13} \mathrm{C} \mathrm{NMR}\left(125 \mathrm{MHz}, \mathrm{CDCl}_{3}\right): \delta 21.3,44.4,44.5,45.1$, 74.4, 74.6, 79.7, 82.5, 121.9, 127.2, 127.4, 127.6, 127.8, $128.2,128.3,128.4,128.5,128.6,129.1,129.2,129.3$, 129.4, 129.5, 131.9, 134.6, 135.2, 138.5; HRMS: calcd. for $\mathrm{C}_{19} \mathrm{H}_{19} \mathrm{BrO}_{2}[\mathrm{M}+\mathrm{Na}]^{+}$381.0466; found, 381.0465.

\subsection{2-(-2-Bromo-3-phenylallyloxy)(4-methoxyphenyl) methyl)oxirane $(\boldsymbol{6 d})$}

Viscous liquid as an inseparable mixture of two isomers in approx. 1:1 ratio. Yield 85\%. IR (Neat): 2997, 1610, 1512, 1249, 1074, 757, $692 \mathrm{~cm}^{-1} ;{ }^{1} \mathrm{H}$ NMR (500 $\left.\mathrm{MHz}, \mathrm{CDCl}_{3}\right): \delta 2.63(\mathrm{dd}, J=2.5,5.0 \mathrm{~Hz}, 0.50 \mathrm{H}$ ), $2.74-2.76(\mathrm{~m}, 1 \mathrm{H}), 2.82(\mathrm{dd}, J=4.0,5.0 \mathrm{~Hz} 0.50 \mathrm{H})$, $3.22-3.24(\mathrm{~m}, 0.50 \mathrm{H}), 3.26-3.28(\mathrm{~m}, 0.50 \mathrm{H}), 3.81(\mathrm{~s}$, $3 \mathrm{H}), 4.18-4.48$ (complex multiplets, $2.50 \mathrm{H}), 4.60$ (d, $J=4.0 \mathrm{~Hz}, 0.50 \mathrm{H}), 6.92(\mathrm{~d}, J=8.0 \mathrm{~Hz}, 2 \mathrm{H}), 7.29-$ 7.34 (m, 6H), 7.41-7.43 (m, 2H); ${ }^{13} \mathrm{C}$ NMR (100 MHz, 
$\left.\mathrm{CDCl}_{3}\right): \delta 44.3,45.2,54.1,55.0,55.3,56.7,56.8,78.9$, 81.4, 84.9, 85.0, 86.4, 86.6, 113.7, 114.0, 114.1, 122.6, $122.7,128.3,128.4,128.5,128.7,129.1,129.2,129.3$, 131.8, 159.9; HRMS: calcd. for $\mathrm{C}_{19} \mathrm{H}_{19} \mathrm{BrO}_{3}[\mathrm{M}+\mathrm{Na}]^{+}$ 397.0415; found: 397.0417 .

\subsection{2-(-2-Bromo-3-phenylallyloxy)(naphthalen-6-yl) methyl)oxirane $(\boldsymbol{6 e})$}

Viscous liquid as an inseparable mixture of two isomers in approx. 2:1 ratio. Yield 89\%. IR (Neat): 3056, 1598, 1488, 1078, 910, $757 \mathrm{~cm}^{-1} ;{ }^{1} \mathrm{H}$ NMR $(500 \mathrm{MHz}$, $\left.\mathrm{CDCl}_{3}\right): \delta 2.72-2.73(\mathrm{~m}, 0.66 \mathrm{H}), 2.74-2.80(\mathrm{~m}, 0.67 \mathrm{H})$, $2.84-2.88(\mathrm{~m}, 0.67 \mathrm{H}), 3.34-3.36(\mathrm{~m}, 0.33 \mathrm{H}), 3.39-3.41$ $(\mathrm{m}, 0.67 \mathrm{H}), 4.27-4.61$ (complex multiplets, $2.67 \mathrm{H})$, $4.84(\mathrm{~d}, J=4.0 \mathrm{~Hz}, 0.33 \mathrm{H}), 7.31-7.33(\mathrm{~m}, 3 \mathrm{H}), 7.43-$ $7.48(\mathrm{~m}, 2 \mathrm{H}), 7.52-7.62(\mathrm{~m}, 3 \mathrm{H}), 7.82-7.87(\mathrm{~m}, 5 \mathrm{H}) ;{ }^{13} \mathrm{C}$ NMR (125 MHz, $\left.\mathrm{CDCl}_{3}\right): \delta 44.4,45.3,54.2,55.0,57.1$, 57.2, 79.7, 82.0, 84.9, 122.6, 124.9, 125.1, 126.3, 126.4, $126.8,127.3,127.8,128.1,128.3,128.5,128.6,131.8$, 133.3, 134.8; HRMS: calcd. for $\mathrm{C}_{22} \mathrm{H}_{19} \mathrm{BrO}_{2}[\mathrm{M}+\mathrm{Na}]^{+}$ 417.0461; found: 417.0464 .

\subsection{2-(-2-Bromo-3-phenylallyloxy)(oxiran-2-yl) methyl)-3a,7a-dihydrobenzo[d][1,3]dioxole (6f)}

Viscous liquid as an inseparable mixture of two isomers in approx. 2:1 ratio. Yield 85\%. IR (Neat): 2995, 1614, 1519, 1249, 1080, 768, $690 \mathrm{~cm}^{-1} ;{ }^{1} \mathrm{H}$ NMR (500 $\left.\mathrm{MHz}, \mathrm{CDCl}_{3}\right): \delta 2.54-2.56(\mathrm{~m}, 0.67 \mathrm{H}), 2.64-2.74(\mathrm{~m}$, $1.33 \mathrm{H}$ ), 3.10-3.16 (m, 1H), 4.09-4.47 (complex multiplets, $2 \mathrm{H}), 5.10-5.12(\mathrm{~m}, 0.67 \mathrm{H}), 5.25-5.29(\mathrm{~m}, 0.33 \mathrm{H})$, 5.88 (2s merged, $2 \mathrm{H}), 6.70-6.79(\mathrm{~m}, 2 \mathrm{H}), 6.83-6.84(\mathrm{~m}$, 1H), 7.16-7.35 (m, 6H), $\left.{ }^{13} \mathrm{C} \mathrm{NMR} \mathrm{(125} \mathrm{MHz,} \mathrm{CDCl}_{3}\right): \delta$ 44.4, 45.3, 54.2, 55.1, 56.8, 75.4, 79.2, 81.5, 84.8, 84.9, $86.6,86.7,107.9,108.0,108.3,108.4,115.1,115.4$, $121.1,121.6,122.6,122.7,126.4,127.8,128.2,128.3$, $128.4,128.5,128.6,129.1,129.2,131.1,131.3,131.8$, 131.9, 140.4, 142.7, 147.9, 148.1; HRMS: calcd. for $\mathrm{C}_{19} \mathrm{H}_{17} \mathrm{BrO}_{4}[\mathrm{M}+\mathrm{Na}]^{+}$411.0208; found: 411.0206.

\subsection{2-(1-(-2-bromo-3-phenylallyloxy)pentyl)oxirane} (6g)

Viscous liquid as an inseparable mixture of two isomers in approx. 4:1 ratio. Yield 84\%. IR (Neat): 2929, 1507, 1490, 1255, 1083, 756, $691 \mathrm{~cm}^{-1} ;{ }^{1} \mathrm{H}$ NMR $(500 \mathrm{MHz}$, $\left.\mathrm{CDCl}_{3}\right): \delta 0.80(\mathrm{t}, J=7.0 \mathrm{~Hz}, 3 \mathrm{H}), 1.17-1.62(\mathrm{~m}, 6 \mathrm{H})$, 2.45 (dd, $J=3.0,4.5 \mathrm{~Hz}, 0.80 \mathrm{H}), 2.70(\mathrm{t}, J=4.5 \mathrm{~Hz}$, $0.80 \mathrm{H}), 2.74(\mathrm{~d}, J=3.5 \mathrm{~Hz}, 0.40 \mathrm{H}), 2.84-2.87$ $(\mathrm{m}, 0.20 \mathrm{H}), 2.92-2.94(\mathrm{~m}, 0.80 \mathrm{H}), 3.12-3.16(\mathrm{~m}$, $0.80 \mathrm{H}), 3.36-3.40(\mathrm{~m}, 0.20 \mathrm{H}), 4.37(\mathrm{~d}, J=4.0 \mathrm{~Hz}$,
$0.20 \mathrm{H}), 4.48\left(\mathrm{AB}_{\mathrm{q}}, \mathrm{J}=15.5 \mathrm{~Hz}, 0.80 \mathrm{H}\right), 7.18-7.23(\mathrm{~m}$, 4H), 7.33-7.37 (m, 2H); ${ }^{13} \mathrm{C} \mathrm{NMR}\left(75 \mathrm{MHz}, \mathrm{CDCl}_{3}\right): \delta$ 14.1, 22.7, 24.9, 25.2, 31.9, 32.0, 32.3, 32.8, 43.3, 45.7, 53.3, 54.8, 58.1, 58.2, 80.2, 85.6, 85.8, 122.9, 128.3, 128.4, 128.5, 131.8; HRMS: calcd. for $\mathrm{C}_{16} \mathrm{H}_{21} \mathrm{BrO}_{2}$ $[\mathrm{M}+\mathrm{Na}]^{+}$347.0623; found: 347.0627 .

\subsection{Typical $\mathrm{Cp}_{2} \mathrm{TiCl}$ mediated addition-elimination} procedure for the synthesis of benzylidene substituted furan derivative $7 a$

A solution of titanocene dichloride (564 mg, 2.28 $\mathrm{mmol})$ in dry THF (10 mL, strictly deoxygenated) was stirred with activated zinc dust $(360 \mathrm{mg}, 5.5 \mathrm{mmol})$ for $1 \mathrm{~h}$ under argon (activated zinc dust was prepared by washing $20 \mathrm{~g}$ of commercially available zinc dust with $60 \mathrm{~mL}$ of $4 \mathrm{M} \mathrm{HCl}$ and thorough washing with water and finally with dry acetone and then dried in vacuum). The resulting green solution was then added dropwise to a stirred solution of the epoxy ether $\mathbf{6 a}$ (345 mg, $1.0 \mathrm{mmol})$ in dry THF (20 mL) at RT under argon during $1 \mathrm{~h}$. The reaction mixture was stirred for 6 hours and was quenched with a saturated solution of sodium dihydrogen phosphate $(5 \mathrm{~mL})$. Most of the solvent was removed under reduced pressure and the residue was extracted with diethyl ether $(4 \times 30 \mathrm{~mL})$. The combined ether layer was washed with saturated $\mathrm{NaHCO}_{3}(2 \times 25 \mathrm{~mL})$ and finally dried over $\mathrm{Na}_{2} \mathrm{SO}_{4}$. After removal of the solvent under reduced pressure the crude residue obtained was purified by column chromatography over silica gel to afford the substituted furan compound (2S,3R)-4-benzylidene-tetrahydro-2phenylfuran-3-yl)methanol ( $E: Z=1: 2)(7 \mathbf{a}, 192 \mathrm{mg}$, $72 \%$ ) as a mixture of two isomers in 1:2 ratio. IR (Neat): 3330, 1598, 1488, 1078, 910, $757 \mathrm{~cm}^{-1} ;{ }^{1} \mathrm{H}$ NMR $\left(500 \mathrm{MHz}, \mathrm{CDCl}_{3}\right): \delta 3.00-3.01(\mathrm{~m}, 0.33 \mathrm{H}), 3.45-3.49$ $(\mathrm{m}, 0.67 \mathrm{H}), 3.76-3.79(\mathrm{~m}, 1 \mathrm{H}), 3.81-3.85(\mathrm{~m}, 0.50 \mathrm{H})$ 3.93-3.97 (m, 0.50H), 4.61-4.95 (complex multiplets, $2.33 \mathrm{H}), 5.22(\mathrm{~d}, J=3.6 \mathrm{~Hz}, 0.67 \mathrm{H}), 6.45(\mathrm{q}, J=2.0$ $\mathrm{Hz}, 0.33 \mathrm{H}), 6.49(\mathrm{~d}, J=2.0 \mathrm{~Hz}, 0.67 \mathrm{H}), 7.16-7.45(\mathrm{~m}$, $10 \mathrm{H}) ;{ }^{13} \mathrm{C}$ NMR $\left(125 \mathrm{MHz}, \mathrm{CDCl}_{3}\right): \delta 52.1,55.9,61.9$, 63.1, 70.3, 73.2, 82.3, 84.6, 122.1, 126.1, 126.3, 127.1, $127.2,127.7,127.9,128.1,128.2,128.6,128.7,136.6$, 136.9, 140.6, 141.4, 141.6; HRMS: calcd. for $\mathrm{C}_{18} \mathrm{H}_{18} \mathrm{O}_{2}$ $[\mathrm{M}+\mathrm{Na}]^{+}$289.1204; found: 289.1205 .

Compounds $\mathbf{7 b}-\mathbf{7 g}$ were prepared following the similar procedure used for the preparation of $\mathbf{7 a}$.

\section{9 (2S,3R)-4-Benzylidene-2-(4-chlorophenyl)} tetrahydrofuran-3-yl)methanol $(E: Z=1: 1)(7 \boldsymbol{b})$

IR (Neat): 3363, 2871, 1596, 1490, 1213, 1062, 825, $756 \mathrm{~cm}^{-1} ;{ }^{1} \mathrm{H}$ NMR $\left(500 \mathrm{MHz}, \mathrm{CDCl}_{3}\right): \delta 2.87(\mathrm{~d}, J=$ 
$5.5 \mathrm{~Hz}, 0.50 \mathrm{H}), 3.33$ (s, 0.50H), 3.64-3.76 (m, $1.50 \mathrm{H})$, $3.86-3.89(\mathrm{~m}, 0.50 \mathrm{H}), 4.56(\mathrm{q}, J=7.5 \mathrm{~Hz}, 1 \mathrm{H}), 4.68-$ 4.86 (complex multiplets, $1.50 \mathrm{H}), 5.12(\mathrm{~d}, J=3.5 \mathrm{~Hz}$, $0.50 \mathrm{H}), 6.37(\mathrm{~d}, J=1.5 \mathrm{~Hz}, 0.50 \mathrm{H}), 6.42(\mathrm{~d}, J=1.0$ $\mathrm{Hz}, 0.50 \mathrm{H}), 7.07-7.31(\mathrm{~m}, 9 \mathrm{H}) ;{ }^{13} \mathrm{C}$ NMR $(125 \mathrm{MHz}$, $\left.\mathrm{CDCl}_{3}\right): \delta 52.1,56.0,61.8,63.0,70.3,73.1,81.6,83.9$, $116.3,122.4,122.5,127.2,127.4,127.5,127.7,128.0$, 128.2, 128.7, 128.8, 133.4, 133.6, 136.4, 136.8, 140.0, 140.8, 141.0; HRMS: calcd. for $\mathrm{C}_{18} \mathrm{H}_{17} \mathrm{ClO}_{2}[\mathrm{M}+\mathrm{Na}]^{+}$ 323.0815; found: 323.0815 .

2.10 (2S,3R)-4-Benzylidene-tetrahydro-2-p-tolylfuran$3-y l)$ methanol $(E: Z=1: 1)(7 c)$

IR (Neat): 3360, 2921, 1598, 1512, 1269, 1176, 813, $696 \mathrm{~cm}^{-1} ;{ }^{1} \mathrm{H}$ NMR $\left(500 \mathrm{MHz}, \mathrm{CDCl}_{3}\right): \delta 2.33$ and $2.34(2 \mathrm{~s}, 3 \mathrm{H}), 2.99(\mathrm{~d}, J=6.0 \mathrm{~Hz}, 0.50 \mathrm{H}), 3.45$ $(\mathrm{d}, J=3.5 \mathrm{~Hz}, 0.50 \mathrm{H}), 3.76(\mathrm{~d}, J=6.0 \mathrm{~Hz}, 0.5 \mathrm{H})$, $3.82(\mathrm{dd}, J=5.0,11.0 \mathrm{~Hz}, 0.50 \mathrm{H}), 3.91-3.97(\mathrm{~m}$, $1 \mathrm{H}), 4.64(\mathrm{ABq}, J=13.0 \mathrm{~Hz}, 1 \mathrm{H}), 4.74-4.93(\mathrm{com}-$ plex multiplets, $1.50 \mathrm{H}), 5.17(\mathrm{~d}, J=3.5 \mathrm{~Hz}, 0.50 \mathrm{H})$, 6.45 (brs, 0.5H), 6.49 (brs, 0.50H), 7.05-7.47 (m, 9H); ${ }^{13} \mathrm{C}$ NMR $\left(75 \mathrm{MHz}, \mathrm{CDCl}_{3}\right): \delta 21.2,52.0,55.8,56.1$, $56.3,61.8,63.0,70.2,73.1,82.2,84.5,109.1,110.5$, $122.0,126.1,126.4,127.1,127.2,128.1,128.2$, 128.7, 129.3, 129.6, 136.6, 137.0, 137.4, 137.7, 138.2, 139.0, 140.8, 141.8; HRMS: calcd. for $\mathrm{C}_{19} \mathrm{H}_{20} \mathrm{O}_{2}[\mathrm{M}+\mathrm{Na}]^{+}$ 303.1361; found: 303.1362 .

2.11 (2S,3R)-4-Benzylidene-tetrahydro-2-(4-methoxyphenyl)(furan3-yl)methanol $(E: Z=1: 2)(7 d)$

IR (Neat): 3417, 2934, 1612, 1513, 1248, 1175, 1033, $756,695 \mathrm{~cm}^{-1} ;{ }^{1} \mathrm{H}$ NMR $\left(500 \mathrm{MHz}, \mathrm{CDCl}_{3}\right): \delta 2.96-$ $3.00(\mathrm{~m}, 0.33 \mathrm{H}), 3.42-3.45(\mathrm{~m}, 0.67 \mathrm{H}), 3.74(\mathrm{~d}, J=$ $6.0 \mathrm{~Hz}, 1 \mathrm{H}), 3.79-3.82(\mathrm{~m}, 3.67 \mathrm{H}), 3.92-3.95(\mathrm{~m}$, $0.33 \mathrm{H}), 4.60(\mathrm{ABq}, \mathrm{J}=13.5 \mathrm{~Hz}, 1 \mathrm{H}), 4.75-4.89(\mathrm{com}-$ plex multiplets, $1.33 \mathrm{H}), 5.14(\mathrm{~d}, J=4.0 \mathrm{~Hz}, 0.67 \mathrm{H})$, $6.45(\mathrm{~d}, J=2.5 \mathrm{~Hz}, 0.33 \mathrm{H}), 6.49(\mathrm{~d}, J=2.5 \mathrm{~Hz}$, 0.67H), 6.86-6.89 (m, 2H), 7.16-7.38 (m, 7H); ${ }^{13} \mathrm{C}$ NMR $\left(75 \mathrm{MHz}, \mathrm{CDCl}_{3}\right): \delta 51.9,55.4,55.6,61.8,63.0$, 70.2, 72.9, 82.1, 84.3, 114.0, 114.1, 121.9, 122.0, 127.1, 127.2, 127.5, 127.8, 128.1. 128.2, 128.7, 133.2, 134.1, 136.6, 137.0, 140.9, 141.9, 159.3, 159.5; HRMS: calcd. for $\mathrm{C}_{19} \mathrm{H}_{20} \mathrm{O}_{3}[\mathrm{M}+\mathrm{Na}]^{+}$319.1305; found: 319.1310 .

2.12 (2S,3R)-4-Benzylidene-tetrahydro-2-(naphthalene2-yl)furan-3-yl)methanol $(E: Z=1: 2)(7 e)$

IR (Neat): 3421, 2939, 1598, 1508, 1062, 819, 752, 696 $\mathrm{cm}^{-1} ;{ }^{1} \mathrm{H}$ NMR $\left(400 \mathrm{MHz}, \mathrm{CDCl}_{3}\right.$ ): $\delta$ 3.09-3.11 (m, $0.33 \mathrm{H}), 3.54-3.58(\mathrm{~m}, 0.67 \mathrm{H}), 3.81-3.89(\mathrm{~m}, 1.50 \mathrm{H})$,
3.98-4.02 (m, 0.50H), 4.67-4.79 (m, 1.33H), 4.82-5.03 $(\mathrm{m}, 0.67 \mathrm{H}), 5.12(\mathrm{~d}, J=6.4 \mathrm{~Hz}, 0.33 \mathrm{H}), 5.39(\mathrm{~d}, J=$ $3.6 \mathrm{~Hz}, 0.67 \mathrm{H}), 6.47(\mathrm{~d}, J=2.4 \mathrm{~Hz}, 0.33 \mathrm{H}), 6.52(\mathrm{~d}$, $J=1.6 \mathrm{~Hz}, 0.67 \mathrm{H}), 7.17-7.52(\mathrm{~m}, 8 \mathrm{H}), 7.80-7.86(\mathrm{~m}$, $4 \mathrm{H}) ;{ }^{13} \mathrm{C}$ NMR $\left(75 \mathrm{MHz}, \mathrm{CDCl}_{3}\right): \delta 52.1,55.9,61.9$, 63.1, 70.4, 73.3, 82.4, 84.7, 122.2, 122.3, 124.2, 124.8, 125.3, 126.0, 126.1, 126.2, 126.3, 127.1, 127.3, 127.7, $127.8,128.1,128.3,128.5,128.6,128.7,133.0,133.4$, 136.5, 137.0, 138.8, 139.5, 140.5, 141.5; HRMS: calcd. for $\mathrm{C}_{22} \mathrm{H}_{20} \mathrm{O}_{2}[\mathrm{M}+\mathrm{Na}]^{+}$339.1356; found: 339.1361 .

\subsection{3 (2S,3R)-2-(Benzo[d][1,3]dioxol-6-yl)} (4-benzylidene-tetrahydrofuran-3-yl)methanol $(E: Z=1: 2)(7 f)$

IR (Neat): 3411, 2885, 1488, 1444, 1247, 1037, 933, 756, $694 \mathrm{~cm}^{-1} ;{ }^{1} \mathrm{H}$ NMR (500 MHz, $\left.\mathrm{CDCl}_{3}\right): \delta 2.86-$ $2.87(\mathrm{~m}, 0.33 \mathrm{H}), 3.32-3.33(\mathrm{~m}, 0.67 \mathrm{H}), 3.63-3.68(\mathrm{~m}$, $1 \mathrm{H}), 3.71-3.74(\mathrm{~m}, 0.50 \mathrm{H}), 3.83-3.87(\mathrm{~m}, 0.50 \mathrm{H}), 4.54$ $(\mathrm{ABq}, J=13.0 \mathrm{~Hz}, 1 \mathrm{H}), 4.64-4.83$ (complex multiplets, $1.33 \mathrm{H}), 5.03(\mathrm{~d}, J=3.5 \mathrm{~Hz}, 0.67 \mathrm{H}), 5.85$ and 5.86 (two singlets, 2H), 6.36 (brs, $0.33 \mathrm{H}$ ), 6.41 (brs, 0.67H), 6.67-6.82 (m, 3H), 7.08-7.30 (m, 5H); ${ }^{13} \mathrm{C}$ NMR $\left(75 \mathrm{MHz}, \mathrm{CDCl}_{3}\right): \delta 52.1,55.7,61.7,62.9$, 70.2, 73.1, 82.2, 84.5, 101.1, 106.7, 106.8, 108.2, 119.6, $120.0,122.1,122.2,127.1,127.3,128.1,128.2,128.7$, 136.1, 136.5, 136.9, 140.5, 141.5, 147.1, 147.4, 148.0; HRMS: calcd. for $\mathrm{C}_{19} \mathrm{H}_{18} \mathrm{O}_{4}[\mathrm{M}+\mathrm{Na}]^{+} 333.1103$; found: 333.1106 .

2.14 (2R,3R)-4-Benzylidene-2-butyl-tetrahydrofuran$3-y l)$ methanol $(E: Z=1: 2)(7 \mathrm{~g})$

IR (Neat): 3414, 2930, 1723, 1449, 1038, 913, 754, $695 \mathrm{~cm}^{-1} ;{ }^{1} \mathrm{H}$ NMR $\left(500 \mathrm{MHz}, \mathrm{CDCl}_{3}\right): \delta$ 0.87-0.89 (m, 3H), 1.22-1.62 (m, 6H), 2.67-2.68 (m, 0.33H), 3.11 (m, 0.67H), 3.61-3.82 (complex multiplets, $2 \mathrm{H}), 3.92$ $(\mathrm{q}, J=5.5 \mathrm{~Hz}, 0.33 \mathrm{H}), 4.16-4.17(\mathrm{~m}, 0.67 \mathrm{H}), 4.43-$ $4.45(\mathrm{~m}, 1.33 \mathrm{H}), 4.64(\mathrm{ABq}, J=14.5 \mathrm{~Hz}, 0.67 \mathrm{H})$, $6.44(\mathrm{~s}, 1 \mathrm{H}), 7.13-7.36(\mathrm{~m}, 5 \mathrm{H}) ;{ }^{13} \mathrm{C}$ NMR $(75 \mathrm{MHz}$, $\left.\mathrm{CDCl}_{3}\right): \delta 14.1,20.6,22.7,25.7,31.9,34.4,34.6$, $49.5,53.3,62.3,64.0,69.0,71.8,80.8,83.3,122.3$, $126.9,127.1,128.0,128.1,128.6,128.7,136.8,137.1$, 141.2, 142.4; HRMS: calcd. for $\mathrm{C}_{16} \mathrm{H}_{22} \mathrm{O}_{2}[\mathrm{M}+\mathrm{Na}]^{+}$ 269.1517; found: 269.1520 .

2.15 Preparation of 1-((Z)-3-(1-(4-methoxyphenyl) allyloxy)-2-bromoprop-1-enyl)benzene (18)

The compound 18 (316 mg, 88\%) was prepared from compound 17 (164 mg, $1.0 \mathrm{mmol}$ ) following the similar procedure used for the preparation of compounds 6a. IR (neat): 2993, 1610, 1512, 1247, 1068, 750, 692 
$\mathrm{cm}^{-1} ;{ }^{1} \mathrm{H}$ NMR $\left(500 \mathrm{MHz}, \mathrm{CDCl}_{3}\right): \delta 3.81(\mathrm{~d}, J=$ $3.0 \mathrm{~Hz}, 3 \mathrm{H}), 4.27-4.39(\mathrm{~m}, 2 \mathrm{H}), 5.05(\mathrm{~d}, J=6.5 \mathrm{~Hz}$, $1 \mathrm{H}), 5.23-5.33(\mathrm{~m}, 2 \mathrm{H}), 5.96-6.03(\mathrm{~m}, 1 \mathrm{H}), 6.90(\mathrm{~d}, J=$ 9.0 Hz, 2H), 7.30-7.36 (m, 6H), 7.43-7.45 (m, 2H); ${ }^{13} \mathrm{C}$ NMR (100 MHz, $\left.\mathrm{CDCl}_{3}\right): \delta 55.4,56.1,73.9,81.0,81.6$, $85.5,86.2,114.0,116.7,116.9,122.6,122.9,128.2$, $128.5,128.7,129.1,131.9,132.3,132.5,135.3,138.4$, 138.6, 159.5; HRMS: calcd. for $\mathrm{C}_{19} \mathrm{H}_{19} \mathrm{BrO}_{2}[\mathrm{M}+\mathrm{Na}]^{+}$ 381.0466; found: 381.0465 .

\subsection{Preparation of the bromo ether $\mathbf{2 4}$}

To a stirred suspension of $\mathrm{NaH}$ (53 mg, 50\% dispersion, $1.1 \mathrm{mmol})$ in dry THF $(1 \mathrm{~mL})$ was added dropwise a solution of alcohol 22a (124 $\mathrm{mg}, 0.50 \mathrm{mmol})$ in dry $\mathrm{THF}(5 \mathrm{~mL})$ at $0^{\circ} \mathrm{C}$ under nitrogen. After the evolution of hydrogen ceased (approx. $25 \mathrm{~min}$ ), a solution of dibromo compound 12 (166 $\mathrm{mg}, 0.60 \mathrm{mmol})$ in dry THF $(7.5 \mathrm{~mL})$ was added dropwise at $0^{\circ} \mathrm{C}$ over $10 \mathrm{~min}$. The reaction mixture was stirred at RT for $3 \mathrm{~h}$ and then carefully quenched with ice water. After removal of most of the solvent under reduced pressure, the resulting residue was extracted with diethyl ether $(4 \times 25 \mathrm{~mL})$. The combined ether extract was successively washed with water $(2 \times 10 \mathrm{~mL})$ and brine $(1 \times 10$ $\mathrm{mL})$ and finally dried $\left(\mathrm{Na}_{2} \mathrm{SO}_{4}\right)$. Solvent was removed under reduced pressure and the crude mass obtained was purified by column chromatography over silica gel (30\% ethyl acetate-petroleum ether) to furnish 24 (209 $\mathrm{mg}, 83 \%)$ as a viscous liquid. $[\alpha]_{\mathrm{D}}{ }^{25}=-156.24(\mathrm{c}=$ 5.0, $\mathrm{CHCl}_{3}$ ); IR (Neat): 2990, 1522, 1250, 1069, 750, $697 \mathrm{~cm}^{-1} ;{ }^{1} \mathrm{H}$ NMR $\left(500 \mathrm{MHz}, \mathrm{CDCl}_{3}\right): \delta$ 1.24-1.70 $(\mathrm{m}, 10 \mathrm{H}), 3.58(\mathrm{dd}, J=7.0,9.0 \mathrm{~Hz}, 1 \mathrm{H}), 3.68(\mathrm{dd}$, $J=6.5,8.5 \mathrm{~Hz}, 1 \mathrm{H}), 3.87-3.88(\mathrm{~m}, 6 \mathrm{H}), 4.16(\mathrm{~d}, J=$ $16 \mathrm{~Hz}, 1 \mathrm{H}), 4.36-4.45(\mathrm{~m}, 2 \mathrm{H}), 4.55(\mathrm{~d}, J=8.0 \mathrm{~Hz}$, $1 \mathrm{H}), 6.83-6.91(\mathrm{~m}, 3 \mathrm{H}), 7.25-7.30(\mathrm{~m}, 4 \mathrm{H}), 7.39-7.41$ $(\mathrm{m}, 2 \mathrm{H}) ;{ }^{13} \mathrm{C}$ NMR $\left(100 \mathrm{MHz}, \mathrm{CDCl}_{3}\right): \delta 23.9,24.1$, 25.2, 35.2, 35.3, 36.4, 55.8, 56.0, 56.6, 65.6, 65.9, 78.6, 78.7, 82.0, 85.3, 86.4, 110.6, 110.7, 110.8, 111.1, 120.5, 120.7, 122.9, 128.1, 128.2, 128.3, 128.4, 128.8, 129.1, 129.8, 130.0, 131.7, 131.8, 149.3, 149.4; HRMS: calcd. for $\mathrm{C}_{26} \mathrm{H}_{31} \mathrm{BrO}_{5}[\mathrm{M}+\mathrm{Na}]^{+}$525.1253; found: 525.1255.

\subsection{Preparation of compound 25}

Compound 24 (503 mg, $1.0 \mathrm{mmol}$ ) was stirred with $80 \%$ aqueous acetic acid $\left(2 \mathrm{~mL}\right.$ ) for $6 \mathrm{~h}$ at $40^{\circ} \mathrm{C}$ (monitored by TLC). After completion of the reaction, the reaction mixture was extracted with ethyl acetate $(3 \times$ $30 \mathrm{~mL}$ ) and the combined organic layer was washed with brine $(5 \mathrm{~mL})$ then dried over $\mathrm{Na}_{2} \mathrm{SO}_{4}$. Solvent was removed under reduced pressure and the residue obtained was purified by column chromatography over silica gel ( $40 \%$ ethyl acetate- petroleum ether) to furnish $25(385 \mathrm{mg}, 91 \%)$ as a viscous liquid. $[\alpha]_{\mathrm{D}}{ }^{25}=$ -133.84 ( $\mathrm{c}=9.2, \mathrm{CHCl}_{3}$ ); IR (neat): $3350,1522,1250$, 1069, 750, $697 \mathrm{~cm}^{-1}$; ${ }^{1} \mathrm{H}$ NMR (400 $\left.\mathrm{MHz}, \mathrm{CDCl}_{3}\right)$ : $\delta 3.37(\mathrm{dd}, J=4.8,11.6 \mathrm{~Hz}, 1 \mathrm{H}), 3.55(\mathrm{dd}, J=3.2$, $11.6 \mathrm{~Hz}, 1 \mathrm{H}), 3.81-3.91(\mathrm{~m}, 7 \mathrm{H}), 4.13(\mathrm{~d}, J=16 \mathrm{~Hz}$, $1 \mathrm{H}), 4.36(\mathrm{~d}, J=16 \mathrm{~Hz}, 1 \mathrm{H}), 4.57(\mathrm{~d}, J=8.0 \mathrm{~Hz}$, $1 \mathrm{H}), 6.83-6.92(\mathrm{~m}, 3 \mathrm{H}), 7.29-7.30(\mathrm{~m}, 4 \mathrm{H}), 7.40-7.42$ $(\mathrm{m}, 2 \mathrm{H}) ;{ }^{13} \mathrm{C}$ NMR $\left(100 \mathrm{MHz}, \mathrm{CDCl}_{3}\right): \delta 56.0,56.7$, 62.6, 75.5, 81.6, 84.8, 86.7, 110.3, 111.2, 120.6, 122.5, 128.4, 128.6, 129.5, 131.8, 149.4; HRMS: calcd. for $\mathrm{C}_{20} \mathrm{H}_{23} \mathrm{BrO}_{5}[\mathrm{M}+\mathrm{Na}]^{+}$445.0627; found: 445.0627.

\subsection{Preparation of compound $\mathbf{2 6}$}

A solution of compound 25 (423 mg, $1.0 \mathrm{mmol}$ ) in DCM $(20 \mathrm{~mL})$ was treated with pyridine $(0.64 \mathrm{~mL}, 8.0$ $\mathrm{mmol}$ ) and $\mathrm{TsCl}(248 \mathrm{mg}, 1.3 \mathrm{mmol})$ was added to it at $0^{\circ} \mathrm{C}$ and then stirred for overnight at RT. The mixture was poured into ice water, the organic layer was separated and the aqueous portion was extracted with DCM $(3 \times 50 \mathrm{~mL})$. The combined organic layers were washed successively with $2 \mathrm{M} \mathrm{HCl}$ solution $(10 \mathrm{~mL})$, water $(10 \mathrm{~mL})$ and brine $(10 \mathrm{~mL})$ and finally dried over $\mathrm{Na}_{2} \mathrm{SO}_{4}$. The solvent was removed under reduced pressure followed by column chromatography over silica gel (25\% ethyl acetate-petroleum ether) to afford the monotosylated derivative $26(514 \mathrm{mg}, 89 \%)$ as a viscous oil. $[\alpha]_{\mathrm{D}}{ }^{25}=-86.49\left(\mathrm{c}=3.4, \mathrm{CHCl}_{3}\right)$; IR (Neat): 3344, 2937, 1595, 1356, 1240, 1069, 754, $698 \mathrm{~cm}^{-1}$; ${ }^{1} \mathrm{H}$ NMR $\left(500 \mathrm{MHz}, \mathrm{CDCl}_{3}\right): \delta 2.32(\mathrm{~s}, 3 \mathrm{H}), 3.72-3.87$ $(\mathrm{m}, 8 \mathrm{H}), 3.94(\mathrm{dd}, J=3.0,10.5 \mathrm{~Hz}, 1 \mathrm{H}), 4.03-4.08$ $(\mathrm{m}, 1 \mathrm{H}), 4.28(\mathrm{~d}, J=15.5 \mathrm{~Hz}, 1 \mathrm{H}), 4.50(\mathrm{~d}, J=$ $7.0 \mathrm{~Hz}, 1 \mathrm{H}), 6.75-6.81(\mathrm{~m}, 3 \mathrm{H}), 7.19-7.25(\mathrm{~m}, 6 \mathrm{H})$, $7.33-7.35(\mathrm{~m}, 2 \mathrm{H}), 7.66(\mathrm{~d}, J=8.0 \mathrm{~Hz}, 2 \mathrm{H}) ;{ }^{13} \mathrm{C}$ NMR $\left(125 \mathrm{MHz}, \mathrm{CDCl}_{3}\right): \delta 21.7,55.9,56.0,56.9,70.0$, 73.3, 80.4, 84.5, 86.9, 110.3, 111.3, 120.4, 122.4, 128.0, 128.4, 128.7, 129.9, 131.7, 131.8, 132.8, 144.9, 149.5; HRMS: calcd. for $\mathrm{C}_{27} \mathrm{H}_{29} \mathrm{BrO}_{7} \mathrm{~S}[\mathrm{M}+\mathrm{Na}]^{+}$599.0715; found: 599.0715 .

\subsection{Preparation of compound 27}

To a stirred suspension of sodium hydride (53 mg, 50\% dispersion, $4.0 \mathrm{mmol})$ in dry THF $(10 \mathrm{~mL})$ at $0^{\circ} \mathrm{C}$ was added dropwise a solution of mono tosylate derivative $26(865 \mathrm{mg}, 1.50 \mathrm{mmol})$ in dry THF $(10 \mathrm{~mL})$ under $\mathrm{N}_{2}$ for $30 \mathrm{~min}$. The reaction mixture was stirred for 1 $\mathrm{h}$ at $0^{\circ} \mathrm{C}$ at RT for $3 \mathrm{~h}$. It was then carefully quenched with ice-water. After removal of most of THF under reduced pressure, the resulting residue was extracted with diethyl ether $(3 \times 50 \mathrm{~mL})$. The combined ether 
extract was washed with water $(10 \mathrm{~mL})$ and brine $(10$ $\mathrm{mL})$ and finally dried $\left(\mathrm{Na}_{2} \mathrm{SO}_{4}\right)$. Removal of the solvent under reduced pressure afforded a viscous liquid which was purified by column chromatography over silica gel (25\% ethyl acetate-petroleum ether) to furnish 27 (510 $\mathrm{mg}, 84 \%)$ as a viscous liquid. $[\alpha]_{\mathrm{D}}{ }^{25}=-125.34(\mathrm{c}=$ 2.6, $\mathrm{CHCl}_{3}$ ); IR (Neat): 2967, 1545, 1389, 1276, 1023, 759, $688 \mathrm{~cm}^{-1} ;{ }^{1} \mathrm{H}$ NMR $\left(500 \mathrm{MHz}, \mathrm{CDCl}_{3}\right): \delta 2.66$ $(\mathrm{dd}, J=3.0,5.0 \mathrm{~Hz}, 1 \mathrm{H}), 2.74-2.84(\mathrm{~m}, 1 \mathrm{H}), 3.23-$ $3.29(\mathrm{~m}, 1 \mathrm{H}), 3.86-3.92(\mathrm{~m}, 6 \mathrm{H}), 4.28-4.32(\mathrm{~m}, 2 \mathrm{H})$, 4.44-4.50 (m, 1H), 6.86-6.98 (m, 3H), 7.28-7.36 (m, $4 \mathrm{H}), 7.41-7.43(\mathrm{~m}, 2 \mathrm{H}) ;{ }^{13} \mathrm{C} \mathrm{NMR}\left(100 \mathrm{MHz}, \mathrm{CDCl}_{3}\right)$ : $\delta$ 44.4, 54.2, 55.1, 56.0, 56.1, 56.9, 81.5, 85.0, 86.5, $110.2,111.2,120.1,120.5,122.7,128.2,128.3,128.4$, 128.5, 129.1, 129.9, 131.9, 149.4; HRMS: calcd. for $\mathrm{C}_{20} \mathrm{H}_{21} \mathrm{BrO}_{4}[\mathrm{M}+\mathrm{Na}]^{+}$427.0521; found: 427.0520 .

\subsection{Preparation of compound $\mathbf{2 8}$}

The compound 28 (246 mg, 73\%) was prepared from compound 27 (400 mg, $0.98 \mathrm{mmol}$ ) by radical cyclization reaction following the similar procedure used for the preparation of compound 7a. The isolated compound was found to be an inseparable mixture of two isomers in a ratio of $1: 1$. IR (neat): $3440,2958,1560$, 1299, 1276, 1033, 750, $678 \mathrm{~cm}^{-1} ;{ }^{1} \mathrm{H}$ NMR (500 $\left.\mathrm{MHz}, \mathrm{CDCl}_{3}\right): \delta 3.00-3.01(\mathrm{~m}, 0.50 \mathrm{H}), 3.45-3.46(\mathrm{~m}$, $0.50 \mathrm{H}$ ), 3.77-3.95 (complex multiplex including several singlets, $8 \mathrm{H}), 4.64(\mathrm{q}, J=13 \mathrm{~Hz}, 1 \mathrm{H}), 4.75-$ 5.08 (complex multiplets, $1.50 \mathrm{H}), 5.13(\mathrm{~d}, J=4.0 \mathrm{~Hz}$, $0.50 \mathrm{H}), 6.47$ (brs, $0.50 \mathrm{H}), 6.53(\mathrm{~d}, J=1.5 \mathrm{~Hz}, 0.50 \mathrm{H})$, 6.84-6.90 (m, 1H), 6.93-6.97 (m, 2H), 7.18-7.40 (m, $5 \mathrm{H}) ;{ }^{13} \mathrm{C} \mathrm{NMR}\left(100 \mathrm{MHz}, \mathrm{CDCl}_{3}\right): \delta$ 51.9, 55.6, 56.0, 56.1, 61.7, 62.8, 70.36, 73.0, 82.3, 84.5, 109.5, 111.2, 118.6, 118.9, 122.0, 122.1, 127.1, 127.3, 128.1, 128.2, 128.7, 133.2, 134.1, 136.6, 137.0, 140.9, 141.9, 159.3, 159.5; HRMS: calcd. for $\mathrm{C}_{20} \mathrm{H}_{22} \mathrm{O}_{4}[\mathrm{M}+\mathrm{Na}]^{+}$ 349.1416; found: 349.1417 .

2.21 Preparation of (Z)-2-bromo-3-(4-methoxyphenyl) prop-2-en-1-ol (35az) and (Z)-2-bromo-3-(3,4-dimethoxyphenyl)prop-2-en-1-ol (35bz)

Dibromo compounds $\mathbf{3 5} \mathbf{a} z$ and $\mathbf{3 5} \mathbf{b} z$ were prepared following the standard literature procedure. ${ }^{18 \mathrm{c}, \mathrm{d}}$

Spectral data of 35bz: IR (neat): 3492, 2933, 1515, 1465, 1271, 1143, 1024, $873 \mathrm{~cm}^{-1}$; ${ }^{1} \mathrm{H}$ NMR $(500 \mathrm{MHz}$, $\left.\mathrm{CDCl}_{3}\right): \delta 3.86(\mathrm{~s}, 6 \mathrm{H}), 4.39(\mathrm{~s}, 2 \mathrm{H}), 6.84(\mathrm{~d}, J=$ $8.5 \mathrm{~Hz}, 1 \mathrm{H}), 6.99(\mathrm{~s}, 1 \mathrm{H}), 7.15(\mathrm{dd}, J=1.0,7.5 \mathrm{~Hz}$, $1 \mathrm{H}), 7.30-7.32(\mathrm{~m}, 1 \mathrm{H}) ;{ }^{13} \mathrm{C} \mathrm{NMR}\left(100 \mathrm{MHz}, \mathrm{CDCl}_{3}\right)$ : $\delta$ 55.9, 69.6, 110.7, 110.8, 122.5, 123.4, 127.5, 127.6,
148.4, 149.0; HRMS: calcd, for $\mathrm{C}_{11} \mathrm{H}_{13} \mathrm{BrO}_{3}[\mathrm{M}+\mathrm{Na}]^{+}$ 294.9946; found: 294.9946.

\subsection{Preparation of 1-((Z)-2,3-dibromoprop-1-enyl)- 4-methoxybenzene (36a)}

A solution of $\mathrm{PBr}_{3}(0.12 \mathrm{~mL}, 1.3 \mathrm{mmol})$ in diethyl ether $(10 \mathrm{~mL})$ was added dropwise to the compound $35 \mathrm{az}$ ( $243 \mathrm{mg}, 1.0 \mathrm{mmol}$ ) at $0^{\circ} \mathrm{C}$ and the solution was stirred for $1 \mathrm{~h}$. After completion of the reaction (monitored by TLC) the solution was then neutralized by aqueous saturated $\mathrm{NaHCO}_{3}$ solution and extracted with dihloromethane $(3 \times 10 \mathrm{~mL})$. The combined organic extract was washed with brine $(10 \mathrm{~mL})$ and dried over anhydrous $\mathrm{Na}_{2} \mathrm{SO}_{4}$. Removal of the solvent under reduced pressure afforded the dibromo compound $\mathbf{3 6 a}$ $(70 \%)$ which was directly used in the next step without any further purification.

2.23 Preparation of 4-((Z)-2,3-dibromoprop-1-enyl)1,2-dimethoxybenzene (36b)

The compound $\mathbf{3 6} \mathbf{b}(72 \%)$ was prepared from $\mathbf{3 5 b z}$ following the same protocol which was used for the preparation of compound $\mathbf{3 6 \mathbf { a }}$ and was directly used in the next step without any further purification.

2.24 Preparation of 2-(((Z)-2-bromo-3-(4-methoxyphenyl)allyloxy)(phenyl)methyl)oxirane (37a)

The compound 37a (292 mg, 78\%) was prepared from 5a (150 mg, $1.0 \mathrm{mmol})$ and 36a (366 mg, $1.2 \mathrm{mmol})$ following the similar procedure used for the preparation of compounds 6a-g. IR (neat): 3001, 1606, 1510, 1249, 1178, 910, $732 \mathrm{~cm}^{-1} ;{ }^{1} \mathrm{H}$ NMR (500 $\mathrm{MHz}, \mathrm{CDCl}_{3}$ ): $\delta 2.89(\mathrm{dd}, J=3.0,5.0 \mathrm{~Hz}, 0.50 \mathrm{H}), 2.98-3.03(\mathrm{~m}$, $1 \mathrm{H}), 3.06(\mathrm{dd}, J=3.5,6.0 \mathrm{~Hz}, 0.50 \mathrm{H}), 3.46-3.47$ $(\mathrm{m}, 0.40 \mathrm{H}), 3.51-3.53(\mathrm{~m}, 0.60 \mathrm{H}), 4.03-4.06(\mathrm{~m}, 3 \mathrm{H})$, 4.41-4.73 (m, 2.70H), $4.87(\mathrm{~d}, J=4.5 \mathrm{~Hz}, 0.30 \mathrm{H})$, 7.06-7.08 (m, 1H), 7.13-7.17 (m, 1H), 7.57-7.67 (m, $7 \mathrm{H})$, 7.84-7.88 (m, 1H); $\left.{ }^{13} \mathrm{C} \mathrm{NMR} \mathrm{(125} \mathrm{MHz,} \mathrm{CDCl}_{3}\right)$ : $\delta 44.3,44.4,45.1,45.3,54.2,55.1,55.2,55.3,57.2$, 74.9, 79.4, 81.8, 82.3, 83.4, 113.6, 114.0, 119.7, 127.2, $127.3,127.4,127.6,127.7,128.6,128.7,128.8,129.0$, 129.8, 130.6. 133.3, 133.4, 137.5, 137.7, 159.6, 159.9; HRMS: calcd. for $\mathrm{C}_{19} \mathrm{H}_{19} \mathrm{BrO}_{3}[\mathrm{M}+\mathrm{Na}]^{+}$397.0415; found: 397.0417.

2.25 Preparation of (4-(4-methoxybenzylidene)-tetrahydro-2-phenylfuran-3-yl) (38a)

The compound 38a (107 mg, 68\%) was prepared from $37 \mathbf{a}(200 \mathrm{mg}, 0.53 \mathrm{mmol})$ as a mixture of two isomers in 
1:1.5 ratio following the similar procedure used for the preparation of compounds 7a-g. IR (neat): 3477,2956 , 1606, 1510, 1251, 1178, 1031, $702 \mathrm{~cm}^{-1}$; ${ }^{1} \mathrm{H}$ NMR $\left(500 \mathrm{MHz}, \mathrm{CDCl}_{3}\right): \delta 2.97-2.98(\mathrm{~m}, 0.40 \mathrm{H}), 3.44(\mathrm{~m}$, $0.60 \mathrm{H}), 3.75-3.82(\mathrm{~m}, 4 \mathrm{H}), 3.93(\mathrm{dd}, J=6.0,11.0 \mathrm{~Hz}$, $1 \mathrm{H}), 4.59-4.67(\mathrm{~m}, 1 \mathrm{H}), 4.73-4.94$ (complex multiplets, $1.40 \mathrm{H}), 5.20(\mathrm{~d}, J=3.5 \mathrm{~Hz}, 0.60 \mathrm{H}), 6.38(\mathrm{~d}, J=2.0$ $\mathrm{Hz}, 0.40 \mathrm{H}), 6.43(\mathrm{~d}, J=1.0 \mathrm{~Hz}, 0.60 \mathrm{H}), 6.84(\mathrm{~d}, J=$ $8.5 \mathrm{~Hz}, 2 \mathrm{H}), 6.91(\mathrm{~d}, J=11.5 \mathrm{~Hz}, 1 \mathrm{H}), 7.10(\mathrm{~d}, J=$ $9 \mathrm{~Hz}, 1 \mathrm{H}), 7.24-7.40(\mathrm{~m}, 5 \mathrm{H}) ;{ }^{13} \mathrm{C}$ NMR $(100 \mathrm{MHz}$, $\left.\mathrm{CDCl}_{3}\right): \delta 52.0,55.3,55.4,55.9,61.9,63.1,70.3,73.2$, $82.3,84.6,114.1,114.2,121.5,121.7,126.1,126.3$, 127.6, 127.9, 128.6, 129.2, 129.3, 129.5, 129.8, 138.4, 139.0, 141.5, 142.2, 158.7; HRMS: calcd. for $\mathrm{C}_{19} \mathrm{H}_{20} \mathrm{O}_{3}$ $[\mathrm{M}+\mathrm{Na}]^{+}$319.1310; found: 319.1312 .

\subsection{Preparation of $(R)-(3,4,5$-trimethoxyphenyl) $((R)$-oxiran-2-yl)methanol (41a)}

Activated powdered $4-\AA$ molecular sieves $(150 \mathrm{mg}$, $25 \mathrm{wt} \%)$ in dry $\mathrm{CH}_{2} \mathrm{Cl}_{2}(5 \mathrm{~mL})$ were placed in a flamedried two-necked round-bottom flask under an argon atmosphere. It was cooled to $-20^{\circ} \mathrm{C}$ and a solution of (-)-DET (104 mg, $0.505 \mathrm{mmol})$ in dry $\mathrm{CH}_{2} \mathrm{Cl}_{2}(2 \mathrm{~mL})$ [previously stirred with $4-\AA$ molecular sieves $(50 \mathrm{mg})$ for $20 \mathrm{~min}$ ] and a solution of $\operatorname{Ti}(i-\mathrm{PrO})_{4}(0.1 \mathrm{~mL}$, $0.337 \mathrm{mmol})$ in dry $\mathrm{CH}_{2} \mathrm{Cl}_{2}(2 \mathrm{~mL})$ [previously stirred with 4 - $\AA$ molecular sieves $(50 \mathrm{mg})$ for $20 \mathrm{~min}$ ] were cannulated sequentially into the reaction flask with stirring. After $20 \mathrm{~min}, 5.5 \mathrm{M} t$-BuOOH in decane $(0.61$ $\mathrm{mL}$ ) was added to the mixture and it was stirred at $-20^{\circ} \mathrm{C}$ for another $0.5 \mathrm{~h}$. Then, a solution of allylic alcohol 40 (760 mg, $3.37 \mathrm{mmol}$ ) in dry $\mathrm{CH}_{2} \mathrm{Cl}_{2}$ (4 mL) [previously stirred with 4 - $\AA$ molecular sieves $(75 \mathrm{mg}$ ) for $20 \mathrm{~min}$ ] was cannulated into the mixture and the stirring was continued for further $4 \mathrm{~h}$. Finally, an aqueous solution of $30 \%$ tartaric acid $(3.4 \mathrm{~mL})$ was added, the mixture was stirred for $0.5 \mathrm{~h}$, and the temperature was allowed to warm to $0^{\circ} \mathrm{C}$. Most of the $\mathrm{CH}_{2} \mathrm{Cl}_{2}$ was removed under reduced pressure and the residue was stirred at $0^{\circ} \mathrm{C}$ for $0.5 \mathrm{~h}$ with $30 \%$ aq $\mathrm{NaOH}(3.5 \mathrm{~mL})$ saturated with $\mathrm{NaCl}$. The resulting mixture was filtered through celite using $\mathrm{Et}_{2} \mathrm{O}$ and the filtrate was placed in a separatory funnel and the organic layer was separated. The aqueous layer was extracted with $\mathrm{Et}_{2} \mathrm{O}$ $(1 \times 30 \mathrm{~mL})$ and the combined ethereal extracts were washed with brine $(30 \mathrm{~mL})$ and dried $\left(\mathrm{Na}_{2} \mathrm{SO}_{4}\right)$. The solvent was removed under reduced pressure and the residue obtained was purified by column chromatography over silica gel (30\% ethyl acetate-light petroleum) to give pure chiral epoxide 41a (347 $\mathrm{mg}, 43 \%$ ). as a colorless viscous liquid. $[\alpha]_{\mathrm{D}}{ }^{25}=-22.8(\mathrm{c}=1$, $\mathrm{CHCl}_{3}$ ); IR (Neat): 3447, 3016, 1541, 1458, 1217,
1130, $756 \mathrm{~cm}^{-1} ;{ }^{1} \mathrm{H}$ NMR (400 MHz, $\mathrm{CDCl}_{3}$ ): $\delta 2.79$ $(\mathrm{t}, J=4.8 \mathrm{~Hz}, 1 \mathrm{H}), 2.95(\mathrm{dd}, J=2.8,4.8 \mathrm{~Hz}, 1 \mathrm{H})$, $3.22(\mathrm{~d}, J=3.2 \mathrm{~Hz}, 1 \mathrm{H}), 3.84(\mathrm{~s}, 3 \mathrm{H}), 3.87(\mathrm{~s}, 6 \mathrm{H})$, $4.83(\mathrm{~d}, J=2.8 \mathrm{~Hz}, 1 \mathrm{H}), 6.62(\mathrm{~s}, 2 \mathrm{H}) ;{ }^{13} \mathrm{C}$ NMR $(125$ $\left.\mathrm{MHz}, \mathrm{CDCl}_{3}\right): \delta$ 43.9, 55.2, 56.3, 61.0, 71.1, 103.4, 135.3, 153.6; HRMS: calcd. for $\mathrm{C}_{12} \mathrm{H}_{16} \mathrm{O}_{5}\left(\mathrm{M}+\mathrm{Na}^{+}\right)$ 263.0895; found: 263.0892 .

2.27 Preparation of $(R)-2-((R)-((Z)-2-$ bromo-3-(3,4dimethoxyphenyl)allyloxy)(3,4,5-trimethoxyphenyl) methyl)oxirane (42a)

Compound 42a (386 mg, 78\%) was prepared from 41a (240 mg, $1.0 \mathrm{mmol})$ and 36b (403 $\mathrm{mg}, 1.2 \mathrm{mmol})$ using the similar procedure used for the preparation of compound 6a. $[\alpha]_{\mathrm{D}}{ }^{25}=-59.2\left(\mathrm{c}=2.81, \mathrm{CHCl}_{3}\right)$; IR (Neat): 3018, 1593, 1463, 1215, 1130, 756, 667 $\mathrm{cm}^{-1} ;{ }^{1} \mathrm{H}$ NMR $\left(400 \mathrm{MHz}, \mathrm{CDCl}_{3}\right): \delta 2.83(\mathrm{dd}, J=$ 2.8, $12.4 \mathrm{~Hz}, 2 \mathrm{H}), 3.19(\mathrm{~m}, 1 \mathrm{H}), 3.81-3.92(\mathrm{~m}, 15 \mathrm{H})$, 4.24-4.45 (m, 3H), 6.59-6.63 (m, 2H), 6.76-6.80 (m, $2 \mathrm{H}), 6.85-6.92(\mathrm{~m}, 1 \mathrm{H}), 7.17(\mathrm{t}, J=3.2 \mathrm{~Hz}, 1 \mathrm{H}) ;{ }^{13} \mathrm{C}$ NMR $\left(125 \mathrm{MHz}, \mathrm{CDCl}_{3}\right): \delta 45.3,45.9,54.1,54.3,54.4$, 55.1, 55.8, 55.9, 56.1, 57.1, 60.4, 60.8, 69.6, 73.6, 75.1, 80.1, 80.4, 80.5, 83.2, 86.7, 104.1, 104.2, 104.4, 110.7, $110.9,111.5,111.7,111.7,112.0,114.4,114.6,121.2$, $121.3,122.4,122.6,122.7,125.1,126.7,126.8,127.4$, 127.5, 128.2, 129.5, 133.0, 133.1, 133.3, 137.0, 137.2, 137.9, 148.5, 148.6, 148.8, 149.0, 149.1, 149.7, 153.3, 153.4, 153.5; HRMS: calcd for $\mathrm{C}_{23} \mathrm{H}_{27} \mathrm{BrO}_{7}[\mathrm{M}+\mathrm{Na}]^{+}$ 517.0838; found: 517.0840 .

\subsection{Synthesis of a mixture of magnofargesin (1) and 7'-epimagnofargesin (2)}

A solution of titanocene dichloride (564 mg, 2.28 $\mathrm{mmol})$ in dry and deoxygenated THF $(10 \mathrm{~mL})$ was stirred with activated zinc dust $(360 \mathrm{mg}, 5.5 \mathrm{mmol})$ for $1 \mathrm{~h}$ under argon (activated zinc dust was prepared by washing $20 \mathrm{~g}$ of commercially available zinc dust with $60 \mathrm{~mL}$ of $4 \mathrm{M} \mathrm{HCl}$ and thorough washing with water and finally with dry acetone and then dried in vacuum). The resulting green solution was then added dropwise to a stirred solution of the epoxy ether 42a (495 mg, 1 $\mathrm{mmol})$ in dry THF $(20 \mathrm{~mL})$ at room temperature under argon during $1 \mathrm{~h}$. The reaction mixture was stirred for 6 $\mathrm{h}$ and was quenched with a saturated solution of sodium dihydrogen phosphate $(5 \mathrm{~mL})$. Most of the solvent was removed under reduced pressure and the residue was extracted with diethyl ether $(4 \times 30 \mathrm{~mL})$. The combined ether layer was washed with saturated $\mathrm{NaHCO}_{3}(2 \times$ $25 \mathrm{~mL}$ ) and finally dried over $\mathrm{Na}_{2} \mathrm{SO}_{4}$. After removal of the solvent under reduced pressure the crude residue obtained was purified by column chromatography 
over silica gel (30\% ethyl acetate-petroleum ether) to afford magnofargesin (1) and 7'-epimagnofargesin (2) as a mixture of two isomers in 1: 1 ratio (324 mg, 78\%). IR (neat): 3442, 3020, 1595, 1494, 1217, 1128, 767, $669 \mathrm{~cm}^{-1} ;{ }^{1} \mathrm{H}$ NMR $\left(400 \mathrm{MHz}, \mathrm{CDCl}_{3}\right): \delta 2.98(\mathrm{~m}$, $0.50 \mathrm{H}, \mathrm{C} 8-H$ for $\mathbf{1}), 3.41$ (m, $0.50 \mathrm{H}, \mathrm{C} 8-H$ for 2$), 3.79$ $3.89\left(\mathrm{~m}, 16 \mathrm{H}, 5 \times \mathrm{OCH}_{3}\right.$ and $\left.\mathrm{C} 9-H\right), 3.97(\mathrm{dd}, J=$ 5.6, $11.2 \mathrm{~Hz}, 1 \mathrm{H}, \mathrm{C} 9-H), 4.63(\mathrm{dd}, J=1.6,14.0 \mathrm{~Hz}$, $1 \mathrm{H}, \mathrm{C} 9^{\prime}-H$ for 2$), 4.73-4.77\left(\mathrm{~m}, 0.50 \mathrm{H}, \mathrm{C}^{\prime}-H\right.$ for $\left.\mathbf{1}\right)$, $4.85(\mathrm{~d}, J=6.4 \mathrm{~Hz}, 0.50 \mathrm{H}, \mathrm{C} 7-H$ for 1$), 4.93(\mathrm{dd}$, $J=1.6,14.0 \mathrm{~Hz}, 0.50 \mathrm{H}, \mathrm{C}^{\prime}-H$ for $\left.\mathbf{1}\right), 5.07(\mathrm{~d}, J=$ $3.6 \mathrm{~Hz}, 0.50 \mathrm{H}, \mathrm{C} 7-H$ for 2$), 6.39(\mathrm{~d}, J=2.0 \mathrm{~Hz}$, $0.50 \mathrm{H}, \mathrm{C}^{\prime}-H$ for 1$), 6.47\left(\mathrm{~d}, J=1.6 \mathrm{~Hz}, 0.50 \mathrm{H}, \mathrm{C7}^{\prime}-\right.$ $H$ for 2), 6.61 (s, $1 \mathrm{H}, \mathrm{C} 2-H$ and $\mathrm{C} 6-H$ for 2$), 6.63$ (s, $1 \mathrm{H}, \mathrm{C} 2-H$ and C6- $H$ for 1$), 6.70-6.74(\mathrm{~m}, 1 \mathrm{H}, \mathrm{Ar} H$ ), 6.81-6.93 (m, 2H, $\mathrm{ArH}) ;{ }^{13} \mathrm{C}$ NMR (75 $\left.\mathrm{MHz}, \mathrm{CDCl}_{3}\right)$ : $\delta$ 52.0, 55.7, 56.0, 56.3, 60.9, 61.8, 62.8, 70.3, 73.1, 82.4, 84.6, 103.1, 103.2, 111.3, 111.6, 120.6, 120.8, $121.8,122.1,129.4,130.0,136.9,137.8,138.8,139.4$, 148.3, 148.4, 149.0, 153.4, 153.5; HRMS: calcd. for $\mathrm{C}_{23} \mathrm{H}_{28} \mathrm{O}_{7}[\mathrm{M}+\mathrm{Na}]^{+} 439.1733$; found: 439.1735 .

The two isomers could not be separated by usual chromatographic methods. But, the spectral and analytical data of the mixture of two isomers were in agreement with the reported values. ${ }^{12,19}$

\section{Results and Discussion}

Thus, the bromo epoxide $\mathbf{6}$ was prepared as an inseparable mixture of two isomers in different ratio from the corresponding aldehyde 3 following standard chemical transformations as shown in scheme 1 . The bromo epoxide 6 on treatment with $\mathrm{Cp}_{2} \mathrm{TiCl}$ in THF under argon afforded the tetrahydrofuran 7 via radical induced cyclization-elimination pathway as an inseparable mixture of cis-trans isomers in different ratio. In some cases, the ratio was found to be 1:1 depending on the substrate. In all cases, radical cyclization of the

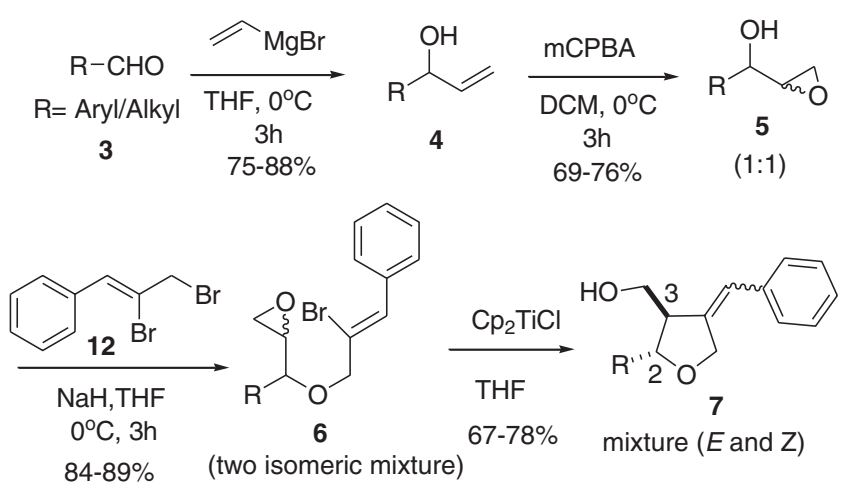

Scheme 1. Radical induced synthesis of tetrahydrofurans. bromo epoxide afforded only the 2,3-trans products as revealed from our earlier studies. ${ }^{11}$

The dibromo compound $\mathbf{1 2}$ was prepared from cinnamaldehyde $\mathbf{8}$ following standard chemical transformations (scheme 2). Thus, a solution of cinnamaldehyde 8 in DCM was stirred for 15 min with $\mathrm{Br}_{2}$ at $0^{\prime} \mathrm{C}$ followed by the addition of $\mathrm{Et}_{3} \mathrm{~N}$ and stirred for $15 \mathrm{~min}$ to yield a 1:10 mixture of $E / Z$ isomers 9 as an yellow oil. After keeping for 3 days at room temperature the mixture of isomers in 9 completely converted to $Z$ - $\alpha$ bromocinnamaldehyde $\mathbf{1 0}$ in the form of a bright yellow solid. Reduction of the aldehyde $\mathbf{1 0}$ with sodium borohydride in the presence of $\mathrm{CeCl}_{3} .7 \mathrm{H}_{2} \mathrm{O}$ furnished the alcohol 11 which was finally brominated using $\mathrm{PBr}_{3}$ to yield the dibromo compound $\mathbf{1 2} .{ }^{13}$

Thus, a series of bromoepoxides were prepared and subjected to radical cyclization using titanocene(III) chloride and the results are summarized in table 1 . The methodology worked well for aromatic (Entry 1-6, table 1) as well as aliphatic substrate (Entry 7, table 1) with comparable yield.

Two possible pathways may be predicted for the formation of the cyclized product 7 as shown in scheme 3 . Path A involves the radical $\mathbf{1 3}$ that undergoes cyclization to furnish the intermediate 14. Then, the expulsion of the bromine radical from $\mathbf{1 4}$ yielded the intermediate $\mathbf{1 5}$ which on acidic work up provided the desired product 7 as an inseparable mixture of two isomers $(E / Z)$. In path $\mathrm{B}$, a diradical species $\mathbf{1 6}$ may be formed which undergoes radical coupling to form the intermediate $\mathbf{1 5}$ and finally to the product $\mathbf{7}$. But, formation of aryl/vinyl radical in path B may be discarded as observed $^{20}$ by Campaña and Cuerva in a control experiment of intramolecular conjugate addition using aryl iodide and $\mathrm{Cp}_{2} \mathrm{TiCl}$.

In support of path $\mathrm{A}$, a separate experiment was carried out where the bromo compound $\mathbf{1 8}$ was treated with $\mathrm{Cp}_{2} \mathrm{TiCl}$ in THF under identical reaction conditions (scheme 4). It was observed that only the unreacted starting bromide $\mathbf{1 8}$ was isolated without a trace of the cyclized product 19 ensuring the inability of $\mathrm{Cp}_{2} \mathrm{TiCl}$ to form a vinyl radical from vinyl bromide.

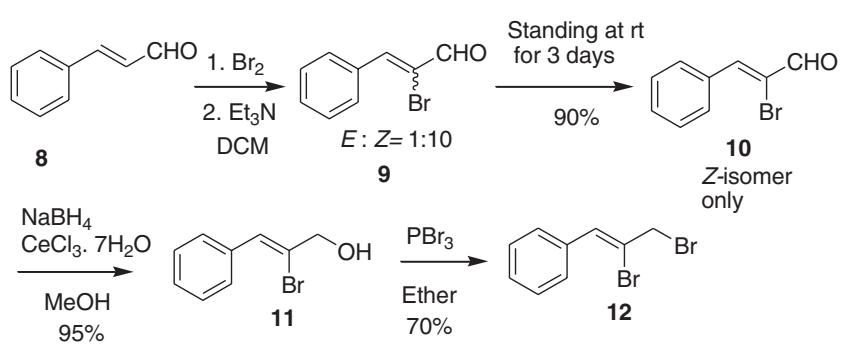

Scheme 2. Synthesis of dibromo compound. 
Table 1. Synthesis of various benzylidene substituted furan moieties.

Sl. No.

Substrate

Product (E:Z)

Yield $(\%)^{\mathrm{a}}$

1<smiles>Br/C(=C\c1ccccc1)COC(c1ccccc1)C1CO1</smiles><smiles>OCC1C(=Cc2ccccc2)CO[C@H]1c1ccccc1</smiles><smiles>Clc1ccc(C(OC/C(Br)=C/c2ccccc2)C2CO2)cc1</smiles>

$7 a(1: 2)$<smiles>Cc1ccc(C(OC/C(=C/c2ccccc2)C2CO2)C2CO2)cc1</smiles><smiles>OCC1C(=Cc2ccccc2)CO[C@H]1c1ccc(Cl)cc1</smiles><smiles>COc1ccc(C(OC/C(Br)=C/c2ccccc2)C2CO2)cc1</smiles><smiles>Cc1ccc([C@@H]2OCC(=Cc3ccccc3)C2CO)cc1</smiles><smiles>O=[W]OC(OCC(Br)=Cc1ccccc1)C1CO1</smiles>

$7 d(1: 2)$<smiles>OCC1C(=Cc2ccccc2)CO[C@H]1c1ccc2ccccc2c1</smiles>

$7 e(1: 2)$

6<smiles>BrC(=Cc1ccccc1)COC(c1ccc2c(c1)OCO2)C1CO1</smiles><smiles>OCC1/C(=C/c2ccccc2)CO[C@H]1c1ccc2c(c1)OCO2</smiles><smiles>CCCCC(OC/C(Br)=C/c1ccccc1)C1CO1</smiles><smiles>CCCC[C@H]1OCC(=Cc2ccccc2)C1CO</smiles>

$7 \mathbf{g}(1: 2)$ 


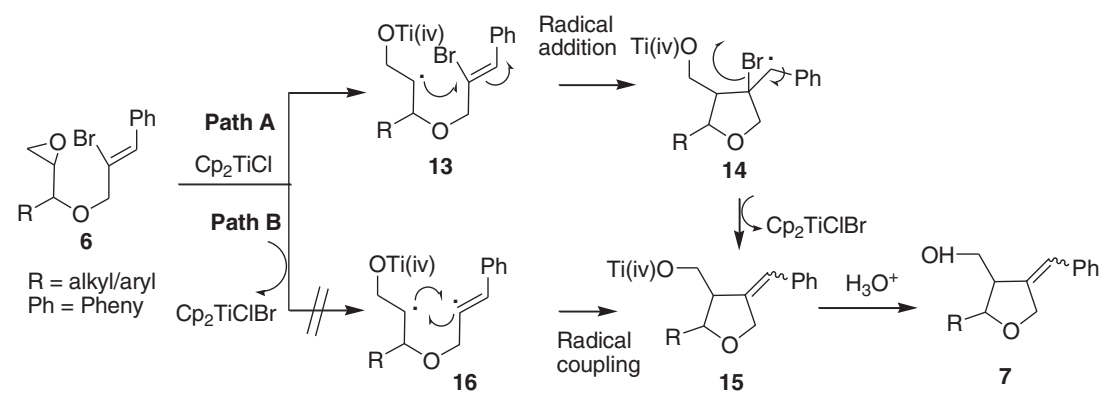

Scheme 3. Probable Mechanism for radical cyclization.

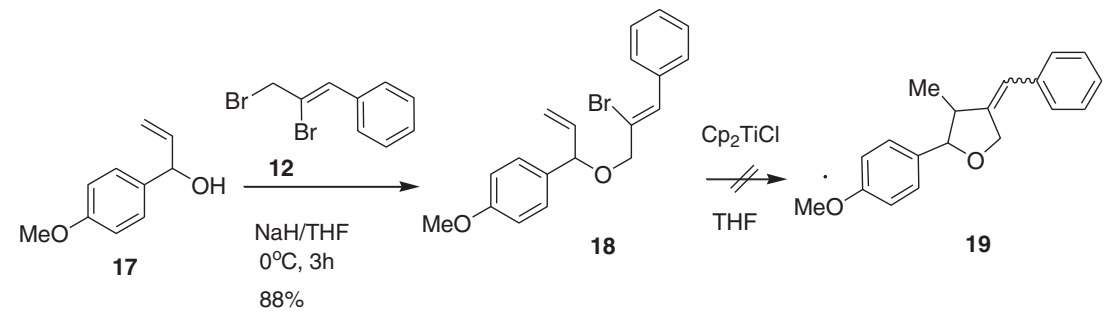

Scheme 4. Experimental support in favour of path A.

For asymmetric synthesis of tetrahydrofurans, easily accessible $R$-2,3- $O$-cyclohexylidine glyceraldehyde 20 was used as a source of chiral pool. Freshly prepared 3,4-dimethoxyphenyl magnesium bromide $\mathbf{2 1}$ was added to the aldehyde $\mathbf{2 0}$ to obtain an inseparable mixture of two isomeric alcohols $\mathbf{2 2} \mathbf{a}$ and $\mathbf{2 2} \mathbf{b}$ in a ratio of 1:1 (scheme 5). The crude mixture of 22 was subjected to PCC oxidation to produce the ketone $\mathbf{2 3}$ in good yield. The ketone 23 was reduced by $\mathrm{LiAlH}_{4}$ in THF at $-78^{\circ} \mathrm{C}$ to afford the known alcohol 22a as the sole product. ${ }^{21 \mathrm{c}}$ The high selectivity of the nucleophilic addition of hydride to carbonyl moiety in $\mathbf{2 3}$ may be explained with the analogy as reported earlier. ${ }^{21}$ The alcohol 22a was then alkylated with dibromo compound 12 in the presence of $\mathrm{NaH}$ in THF under argon to furnish 24. The aryl ether $\mathbf{2 4}$ on treatment with $80 \%$
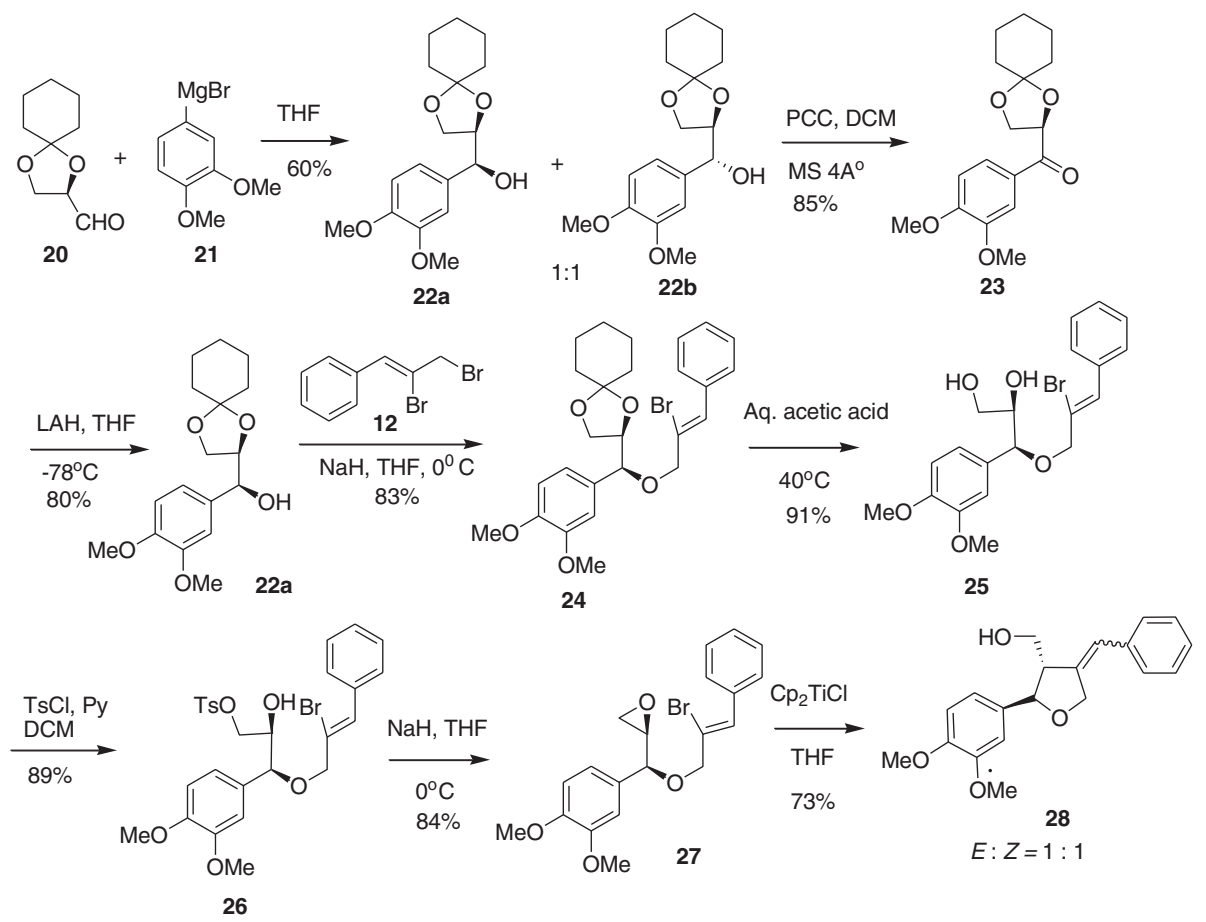

Scheme 5. Asymmetric synthesis of tetrahydrofurans. 
aqueous acetic acid at $40^{\circ} \mathrm{C}$ afforded the diol 25 . The diol 25 was selectively mono-tosylated using tosyl chloride with excess of pyridine in DCM to furnish the mono-tosylated alcohol 26 which on treatment with $\mathrm{NaH}$ in THF produced the chiral epoxide $\mathbf{2 7}$ in $84 \%$ yield. The chiral radical precursor 27 on treatment with $\mathrm{Cp}_{2} \mathrm{TiCl}$ in $\mathrm{THF}$ under argon produced the cyclized product $\mathbf{2 8}$ as an inseparable mixture of two isomers in equal ratio $(E: Z=1: 1)$.

To study the scope of the method an attempt to prepare substituted dibromo compound 36a,b following the procedure as stated in scheme 2 was unsuccessful as it produced only a mixture of unidentified products. Finally, the aromatic substituted dibromo compounds 36a and 36b were prepared following the procedure as depicted in scheme 6 .

Thus, phosphonium ylide $31^{18 a, b}$ prepared from bromophoshphonium salt $\mathbf{3 0}$ which was selectively brominated following standard literature method ${ }^{18 c}$ to form bromo carbethoxymethylenetriphenylphosphorane $\mathbf{3 2}$. The bromide $\mathbf{3 2}$ was refluxed separately with aldehyde 33a and 33b to furnish the isomeric unsaturated bromoesters 34a and $\mathbf{3 4 b}$ respectively $(E / Z=1: 10)$. Without further purification, the mixture of esters in $\mathbf{3 4 a}$ and 34b was separately treated with DIBAL-H in THF at $-78^{\circ} \mathrm{C}$ to produce the corresponding isomeric mixture of bromo alcohols $\mathbf{3 5} \mathbf{a}$ and $\mathbf{3 5} \mathbf{b}$ in $1: 10$ ratio $(E / Z)$. The major $Z$-isomer in 35az (76\%) and $\mathbf{3 5 b z}(75 \%)$ was separated by column chromatography over silica gel. ${ }^{18 \mathrm{~d}}$ Alcohols $\mathbf{3 5 a} z$ and $\mathbf{3 5} \mathbf{b} z$ were brominated separately with $\mathrm{PBr}_{3}$ to produce the corresponding methoxy substituted dibromo compounds 36a (64\%) and 36b (62\%). Alkylation of $\mathbf{5 a}$ with the dibromo compound 36a furnished the epoxy ether 37a. The epoxy ether 37a was then treated with $\mathrm{Cp}_{2} \mathrm{TiCl}$ in THF under argon to furnish the furan moiety 38a in 68\% yield (scheme 7). The result showed no significant change in the diastereomeric ratio $(E / Z=1: 1.5)$ which implied that the electronic effect of the methoxy group in the aromatic moiety failed to incorporate much selectivity.

We then turned our attention to the synthesis of furano lignans using the similar protocol. Lignans have attracted much interest over last few decades on account of their widespread occurrence in nature ${ }^{22}$ and broad range of biological activities. ${ }^{19,23-27}$ Magnofargesin, an antagonist of platelet-activity factor $(\mathrm{PAF})^{28}$ is a class of lignan and has been a long standing interest for synthetic chemists. Although plentiful synthetic

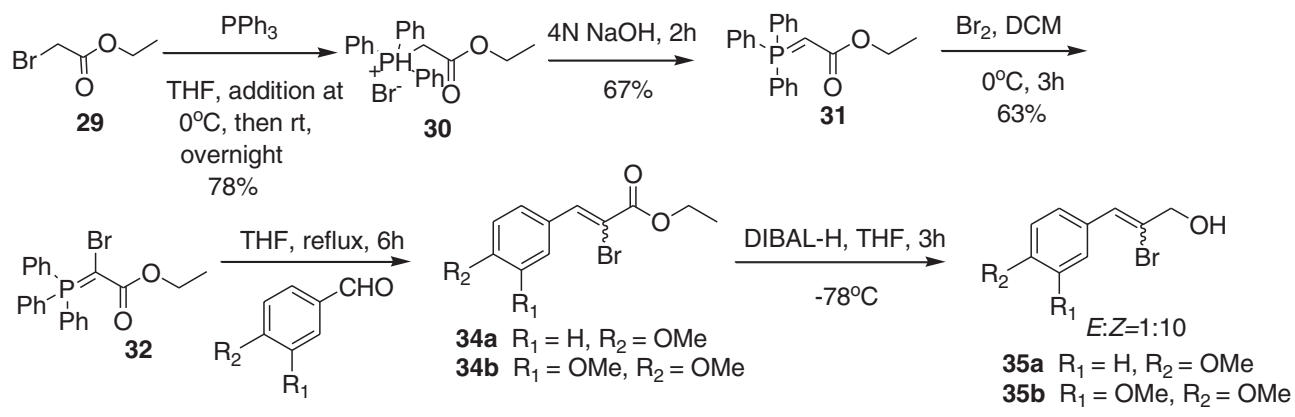

33a $\mathrm{R}_{1}=\mathrm{H}, \mathrm{R}_{2}=\mathrm{OMe}$

33b $\mathrm{R}_{1}=\mathrm{OMe}, \mathrm{R}_{2}=\mathrm{OMe}$

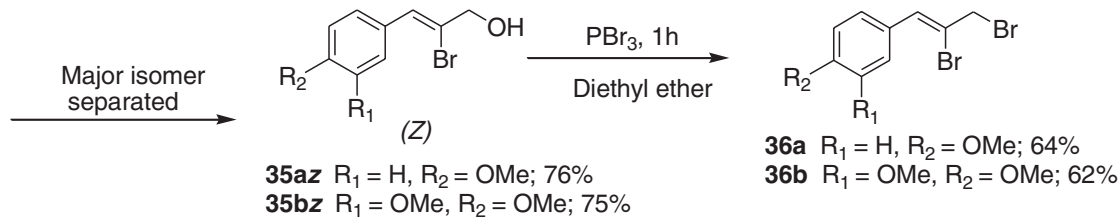

Scheme 6. Synthesis of aromatic substituted dibromides.

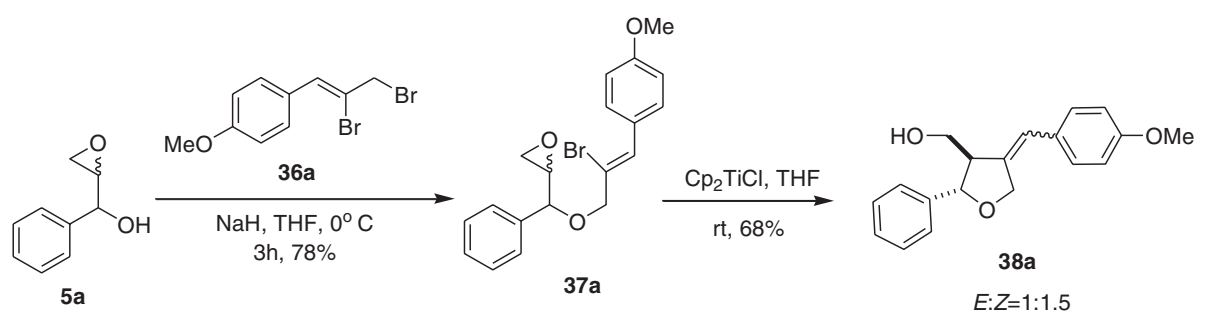

Scheme 7. Synthesis of tetrahydrofurans. 

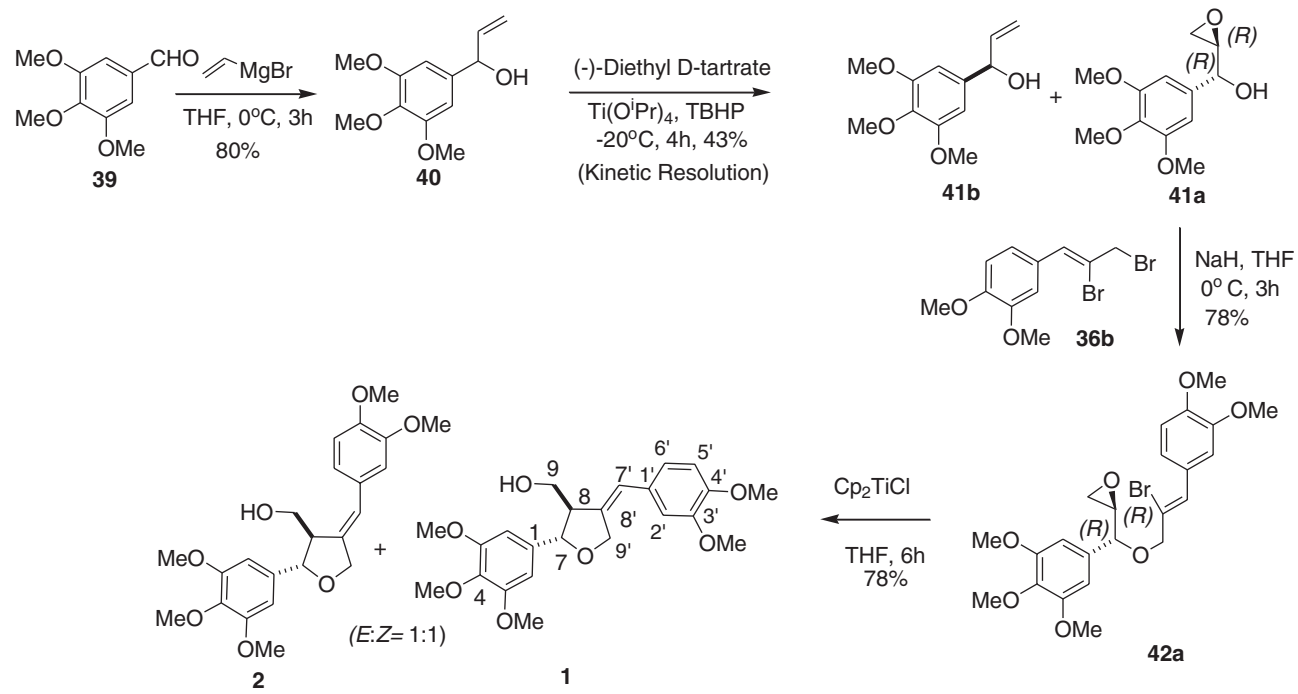

Scheme 8. Formal synthesis of magnofargesin and $7^{\prime}$-epimagnofargesin.

strategies leading to benzyledene substituted tetrahydrofurans have been reported in the literature, the major drawbacks are tedious reaction procedures, low yield and tedious separation technique. ${ }^{29}$ In continuation of our study on $\mathrm{Cp}_{2} \mathrm{TiCl}$ mediated radical induced synthesis of natural product, we demonstrated the formal synthesis of magnofargesin (1) and its stereoisomer epi-magnofargesin (2) ${ }^{11}$ in optically active forms using addition-elimination methodology. Thus, 3,4,5trimethoxybenzaldehyde $\mathbf{3 9}$ was treated with vinyl magnesium bromide to furnish the allyl alcohol $\mathbf{4 0}$ which on Sharpless kinetic resolution, ${ }^{14}$ using (-)diethyl tartrate, titanium(IV) isopropoxide $\left[\mathrm{Ti}^{i}(\mathrm{PrO})_{4}\right]$, tert-butyl hydroperoxide and $4-\AA$ molecular sieves in $\mathrm{DCM}$ at $-20^{\circ} \mathrm{C}$ afforded the chiral epoxy alcohol 41a in $43 \%$ isolated yield (95\% ee, determined from the corresponding Mosher ester) ${ }^{14 \mathrm{c}}$ (scheme 8). The other enantiomer of the allylic alcohol $\mathbf{4 1 b}$ was isolated as such.

The pure epoxy alcohol 41a was then alkylated using the dibromo compound 36b in the presence of $\mathrm{NaH}$ in THF to furnish the chiral epoxy ether 42a in good yield. Finally, the chiral radical precursor $\mathbf{4 2 a}$ when treated with $\mathrm{Cp}_{2} \mathrm{TiCl}$ in THF under argon afforded a mixture of $\mathbf{1}$ and 2 in a ratio of 1:1. The ratio of the two isomers was determined from the ${ }^{1} \mathrm{H}$ NMR spectrum of the crude cyclized product and compared with the values reported in the literature. ${ }^{11,29}$ Since, the total synthesis of $\mathbf{1}$ and $\mathbf{2}$ has been reported by Wardrop ${ }^{29}$ by separating two isomers using special technique, we accomplished the formal synthesis of two naturally occurring furano lignans, magnofargesin (1) and 7'-epimagnofargesin (2) in optically active forms through $\mathrm{Cp}_{2} \mathrm{TiCl}$ mediated radical induced addition-elimination pathway.

\section{Conclusions}

In conclusion, we have successfully developed a simple and efficient $\mathrm{Cp}_{2} \mathrm{TiCl}$ mediated radical induced synthetic protocol for the synthesis of benzylidene substituted tetrahydrofurans following the additionelimination strategy. The technique has been applied to the total synthesis of a mixture of naturally occurring furano lignans, magnofargesin and its epimer 7'-epimagnofargesin, through addition-elimination process. Since, magnofargesin and its epimer 7 '-epimagnofargesin have already been separated earlier from the mixture by Wardrop using special technique, we acomplished the formal synthesis of two naturally occurring furano lignans, magnofargesin (1) and 7'epimagnofargesin (2) in optically active forms.

\section{Supplementary Information (SI)}

Copies of NMR spectra of unknown compounds are available in Supplementary Information at www.ias.ac. in/chemsci.

\section{Acknowledgements}

P. C. thanks CSIR, New Delhi for awarding research fellowship (No. 9/80(677)2009-EMR-I).

\section{References}

1. Kharasch M S, Jerome J J and Urry W H $1950 \mathrm{~J}$. Org. Chem. 15966

2. Heiba E I and Dessau R M 1967 J. Am. Chem. Soc. 893772

3. Baldwin J E, Kelly D R and Ziegler C B 1984 J. Chem. Soc. Chem. Commun. 133 
4. Harris F L and Weiler L 1987 Tetrahedron Lett. 282941

5. Patel V F and Pattendon G 1987 J. Chem. Soc., Chem. Commun. 871

6. Bosch E and Bachi M D 1993 J. Org. Chem. 585581

7. (a) Miyata O, Ozawa Y, Ninomiya I and Naito T 2000 Tetrahedron 56 6199; (b) Miyata O, Shirai A, Yoshino S, Takeda Y, Sugiura M and Naito T 2006 Synlett 893

8. (a) Brioche J, Michalak M, Quiclet-Sire B and Zard S Z 2011 Org. Lett. 13 6296; (b) Zhang H, Hay E B, Geib S J and Curran D P 2013 J. Am. Chem. Soc. 13516610

9. (a) Stanislawski P C, Willis A C and Banwell M G 2007 Org. Lett. 8 2143; (b) Banwell M G, Kokas O J and Willis A C 2007 Org. Lett. 83503

10. (a) Apte S, Radetich B, Shin S and Rajanbabu T V 2004 Org. Lett. 6 4053; (b) Lowinger T B and Weiler L 1990 Can. J. Chem. 681636

11. (a) Mandal S K, Paira M and Roy S C 2010 J. Chem. Sci. 122 423; (b) Saha S, Chakraborty P and Roy S C 2014 J. Chem. Sci. 126 837; (c) Chakraborty P, Jana S, Saha S and Roy S C 2012 Tetrahedron Lett. 536584

12. Rajan Babu T V and Nugent W A 1994 J. Am. Chem. Soc. 116986 and references cited therein.

13. (a) Gilley C B, Buller M J and Kobayashi Y 2007 Org. Lett. 9 363; (b) Bowman W R, Bridge C F, Brookes P, Cloonan M O and Leach D C 2002 J. Chem. Soc., Perkin Trans. 158

14. (a) Gao Y, Hanson R M, Klunder J M, Ko S Y, Masamune H and Sharpless K B 1987 J. Am. Chem. Soc. 1095765 and references cited therein; (b) Procter G 1996 In Asymmetric Synthesis (New York: Oxford University Press) p. 178; (c) Banerjee B and Roy S C 2005 Synthesis 2913; (d) Fang B, Xie X, Li H, Jing P, Gu J and She X 2013 Tetrahedron Lett. 546349

15. Logan A W J, Parker J S, Hallside M S and Burton J W 2012 Org. Lett. 142940

16. Bouziane A, Hélou M, Carboni B, Carreaux F, Demerseman B, Bruneau C and Renaud J-L 2008 Chem. Eur. J. 145630

17. Midland M M and Koops R W 1990 J. Org. Chem. 55 5058

18. (a) Werkhoven $\mathrm{T}$ M, van Nispen R and Lugtenburg J 1999 Eur. J. Org. Chem. 2909; (b) Mao Y and Mathey F 2012 Org. Lett. 14 1162; (c) Gong X, Yang H, Liu H, Jiang Y, Zhao Y and Fu H 2010 Org. Lett. 12 3128; (d) Ohno H, Iuchi M, Kojima N, Yoshimitsu T, Fujii N and Tanaka T 2012 Chem. Eur. J. 185352
19. Nitao J K, Johnson K S, Scriber J M and Nair M G 1992 J. Chem. Ecol. 181661

20. Máquez I R, Miguel D, Millán A, Marcos M L, Cienfuegos L Á d, Campaña A G and Cuerva J M 2014 J. Org. Chem. 791529

21. (a) Chikashita H, Nikaya T, Uemura $H$ and Itoh $\mathrm{K}$ 1989 Bull. Chem. Soc. Jpn. 62 2121; (b) Mulzer J and Angermann A 1983 Tetrahedron Lett. 24 2843; (c) Saha S, Chakraborty P and Roy S C 2014 J. Chem. Sci. 126837

22. (a) C B S Rao (Ed.) 1978 The Chemistry of Lignans (Waltair: Andhra University Press); (b) Whiting D A 1985 Nat. Prod. Rep. 2 191; 19874 499; 19907 349; (c) Ward R S Nat. Prod. Rep. 199310 1; 199512183

23. (a) O R Gottlieb 1977 In New Natural Products and Plant Drugs with Pharmacological, Biological or Therapeutical Activity (Berlin-Heidelberg: SpringerVerlag) p. 227; (b) MacRae W D and Towers G H N 1984 Phytochemistry 231207 and references cited therein.

24. (a) Keller-Juslen C, Kuhn M, Von Warburg A and Stahelin H 1971 J. Med. Chem. 14 936; (b) Kupchan, S M, Britton R W, Ziegler M F, Gilmore C J, Restivo R J and Bryan R F 1973 J. Am. Chem. Soc. 95 1335; (c) Weiss S G, Tin-Wa M, Perdue R E and Farnsworth N R 1975 J. Pharm. Sci. 64 95; (d) Hartwell J L 1976 Cancer Treat. Rep. 60 1031; (e) Carter S K and Livingston R B 1976 Cancer Treat. Rep. 601141

25. (a) Kamikado T, Chang C F, Sakurai S A and Tamura S 1975 Agric. Biol. Chem. (Jpn.) 39 833; (b) Russell G B, Singh P and Fenemore P G 1976 Aust. J. Biol. Sci. 2999

26. Richardson M D, Peterson J R and Clark A M 1992 Phytother. Res. 6274

27. (a) Yamauchi S and Taniguchi E 1991 Agric. Biol. Chem. 55 3075; (b) Yamauchi S and Tanighchi E 1992 Biosci. Biotech. Biochem. 56 412; (c) Yamauchi S, Nagata S and Taniguchi E 1992 Biosci. Biotech. Biochem. 56 1193; (d) Yamauchi S and Taniguchi E 1992 Biosci. Biotech. Biochem. 561744

28. (a) Miyazawa M, Hiroyuki K and Kameoka H 1996 Phytochemistry 42 531; (b) Miyazawa M, Kasahara H and Kameoka H 1995 Nat. Prod. Lett. 7 205; (c) Myazawa M 1994 Chem. Abstr. 12191784

29. Wardrop D J and Fritz J 2006 Org. Lett. 83659 